\title{
Rursus
}

Russus

Poiétique, réception et réécriture des textes antiques

$4 \mid 2009$

Erotica

\section{Les citations dans le Dialogue sur l'amour (Eroticos logos) de Plutarque}

Arnaud Zucker

\section{OpenEdition}

Journals

Édition électronique

URL : http://journals.openedition.org/rursus/312

DOI : $10.4000 /$ rursus.312

ISSN : 1951-669X

Éditeur

Université Nice-Sophia Antipolis

Référence électronique

Arnaud Zucker, «Les citations dans le Dialogue sur l'amour (Eroticos logos) de Plutarque », Rursus [En ligne], 4 | 2009, mis en ligne le 18 février 2009, consulté le 30 avril 2019. URL : http:// journals.openedition.org/rursus/312; DOI : 10.4000/rursus.312

Ce document a été généré automatiquement le 30 avril 2019.

Rursus 


\title{
Les citations dans le Dialogue sur l'amour (Eroticos logos) de Plutarque
}

\author{
Arnaud Zucker
}

\section{Introduction}

1 La citation, en dépit des définitions normatives et des travaux récents, n'est pas un type de discours clair (DARBO-PESCHANSKI 2006). A la fois énoncé (répété) et énonciation (répétante), selon la formule d'A. Compagnon (1979: 56) elle joue un rôle fondamental et complexe dans la littérature antique. A partir des Alexandrins (III ${ }^{\mathrm{èm}}$ s. av. J.-C.), les littérateurs, savants et poètes, philosophes et sophistes, se perçoivent essentiellement comme des héritiers. La pratique citationnelle constitue alors une expression essentielle de cette identité intellectuelle acquise et d'un capital symbolique partagé. Elle est, a priori, suffisamment justifiée, quel qu'en soit le cadre, par cette fonction culturelle d'allégeance et d'affiliation.

2 Mais il ne s'agit pas, naturellement, d'un marquage abstrait ou rituel, et les citations assument, textuellement, un rôle plus ou moins structurant dans le discours et l'argumentation. La fréquence des citations dans les textes de la période « romaine » de la littérature grecque est un trait majeur de celle-ci et elles fournissent parfois, par leur entrelacs, la trame même des œuvres. Plutarque, exemplaire par l'étendue de sa culture et la qualité de sa formation est sûrement « einer der Zitatenfreundigsten » (ZIEGLER 1951 : 914).

\section{Pratique}

\subsection{Extension de la citation}

Il est toutefois difficile d'isoler cette pratique ou d'en établir une typologie convenable. Les modes de l'intertextualité ne constituent pas des options distinctes et se présentent d'avantage sous la forme d'une scala poetica, sur laquelle on passe le plus souvent 
insensiblement d'un type à un autre, de la suggestion à la reprise infléchie, de la paraphrase au commentaire, etc. En témoigne la grande difficulté que nous avons à démarquer dans la littérature grecque deux opérations qui relèvent pour nous a priori de

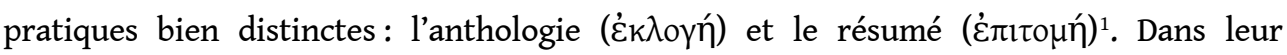
répertoire des citations plutarquéennes, W. Helmbold \& E. O’Neil (1959 : VIII), distinguent quatre paliers : la citation (idéalement littérale), la référence (allusion à un auteur), la réminiscence (imitation inconsciente), et la paraphrase (reprise de thèmes, parfois aussi d'exemples). A. Van Den Hoek, analysant les techniques citationnelles de Clément, autre maniaque du genre $^{2}$, reprend et se propose d'expliciter la typologie précédente. Elle aboutit à une distinction qui, malgré ou à cause de sa souplesse lucide, s'avère elle-même peu praticable. C'est précisément la nature au fond quantitative ${ }^{3}$, et non qualitative, de ce classement qui l'a séduite et qu'elle maintient dans une autre typologie, sur laquelle elle arrête son choix, et s'offre comme une échelle de $\mathrm{A}$ à $\mathrm{D}$ : «A meant a certain dependence (which in the other terminology was a quotation or paraphrase); $B$ was probable dependence (paraphrase or reminiscence); $C$ unprovable dependence (reminiscence), $D$ no dependence » (VAN DEN HOEK $1996: 229$ ).

4 Etudiant l'œuvre d'un contemporain de Plutarque, Lucien de Samosate, J. Bompaire se voit amené à réfléchir à la pulsion citationnelle de Lucien, mais la définition qu'il propose, en revanche, faite de préjugés modernes et d'embarras méthodologique, la confond presque, de manière très lucianesque, avec la littérature tout court: « reproduction immédiate d'un texte (directe ou paraphrasée), allusion reconnaissable à un passage ou même à une œuvre, et à un degré plus éloigné encore la réminiscence, à condition qu'elle ne soit pas équivoque » (BOMPAIRE 1958 : 362). Cette dilution témoigne, en effet, d'une imprégnation profonde de la théorie antique de la création que des modernes savent parfois gloser : «Ecrire, car c'est toujours récrire, ne diffère pas de citer " (COMPAGNON 1979: 34). Le danger inverse d'une théorie de la citation est de suggérer l'existence, dans tout texte, de parties étrangères, formant un dépôt exo-textuel. Hors peut-être en quelques œuvres qui poussent à la limite le procédé (et qui sont à ce titre nécessairement méta-littéraires), la citation ne compromet pas l'unité du texte, elle tendrait plutôt à l'augmenter en vertu de la formule de l'addition créative : $1+1=3$. La citation, soluble dans le texte, est soumise à une passion ambivalente de l'auteur, partagé entre deux tendances : exhiber et incorporer. Mais cette dualité est moins une alternative qu'une complicité.

5 Le lecteur doit en tout cas se déprendre de l'idée pauvre, naïve et séduisante que la citation pourrait constituer en littérature une simple illustration ornementale (вомРАIRE 1958 : 379). Sans contester que ce goût puisse être une caractéristique du style antique, surtout de l'époque hellénistique et romaine, on doit rejeter une telle qualification, car elle se donne pour une explication suffisante, dissuadant de ce fait toute autre forme de procès. La raison d'être d'une citation touche non seulement au style, qui n'est rien moins qu'un « ornement ", mais aussi à la composition, à l'écriture et à la communication. Ces précédentes mains participent à la génération d'un texte authentique : elles ne constituent pas une décoration luxueuse mais un repère intellectuel et parfois un point de départ, comme des bouées qui ponctuent une étape de natation.

6 L'inventaire des citations dans une œuvre pose divers problèmes non seulement définitionnels, mais aussi méthodologiques, car le terme de "citation» désigne davantage des théories modernes qu'un type universel d'énonciation. Ce n'est pas un concept grec et il n'y a pas, pour la « citation antique », de miroir terminologique (NICOLAS 
2006 : 10). On devrait, par ailleurs, s'interdire, le plus souvent, d'identifier une citation littérale (ou au contraire déformée) en raison du caractère définitivement lacunaire de notre information sur les leçons des manuscrits et notre ignorance de la bibliothèque précise des auteurs anciens. Notre conception moderne, très " littéraliste ", du texte, qui distingue radicalement discours " direct » et discours « indirect », et rigoureusement la reprise littérale d'un passage et sa reformulation, nous conduit, dans l'étude des textes anciens, au désespoir ou à la résipiscence. Non seulement parce que cette évaluation est rendue impossible par notre documentation, mais parce que ce principal clivage qui confond 'identique' et 'littéral', est passablement impertinent aux yeux des Grecs.

7 Est-ce qu'un écrivain grec place sa fidélité à un modèle ou à sa pensée dans la reprise

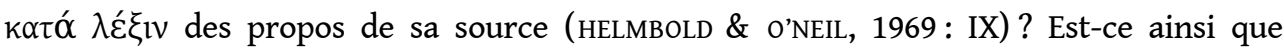
Plutarque marque et paye sa dette? Les études critiques sur les «techniques» de la citation chez un auteur ancien aboutissent régulièrement au même constat d'une grand variété des pratiques. Etudiant les citations qu'il fait d'Hérodote, D. Lenfant (1999: 114) conclut que «Plutarque va de la reprise littérale, à la paraphrase et au remaniement camouflé ». L'usage des citations comme les principes gréco-latins de la traduction montrent, au-delà des variations théoriques, que la lettre compte toujours moins que le sens, le verbum, moins que le sensus 4 . Si les citations poétiques sont in litteris mieux "préservées", il faut y voir davantage un effet de la métrique qu'une révérence particulière pour la forme versifiée, qui ne semble pas jouir d'un statut supérieur qui exigerait un traitement de faveur. Il en résulte néanmoins que les citations prosaïques sont susceptibles d'échapper plus fréquemment au repérage du critique.

\subsection{Silence des rhétoriciens}

8 Des auteurs comme Lucien, Aristide, Clément d'Alexandrie font dans leur œuvre plusieurs milliers de citations. Cette sollicitation des maîtres de vérité peut même se développer, sinon comme genre, du moins comme exercice, dans des sortes de chants amébées sous forme de joutes citationnelles 5 . Bien qu'elle soit un aspect fondamental de la mimesis et de la paideia (CRIBIORE 1994) cette pratique n'est pas abordée dans les manuels de rhétorique et seulement perceptible dans une terminologie vague composée de mots parfois rares,

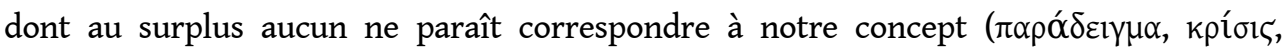

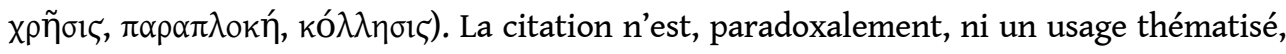
ni une pratique institutionnelle (COMPAGNON 1979: 95-96; GANGLOFF 2006: 101-102). Pourtant sa fonction rhétorique est évidente et son poids considérable dans la formation à l'écriture et la création littéraire, comme à la construction de soi ${ }^{6}$. Les archéologues de la théorie de la citation s'appuient, traditionnellement et presque exclusivement, sur Quintilien ${ }^{7}$. Avant les manuels des Rhetores, Aristote (Rhétorique 1.15, $1375 \mathrm{~b} 28$ sq.) signale la citation comme un outil de la rhétorique, et elle s'apparente, comme parole d'autorité invoquée, à la sentence, à la maxime ou au proverbe ( $\gamma v \tilde{\omega} \mu \alpha \iota$ ou $\left.\pi \alpha \rho \circ \mu_{1} \alpha_{\alpha}\right)$; c'est, dans la Rhétorique d'Aristote, le seul cadre qui permet de l'évoquer (COMPAGNON 1979 : 127-130);

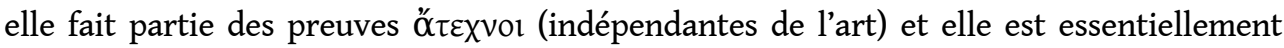

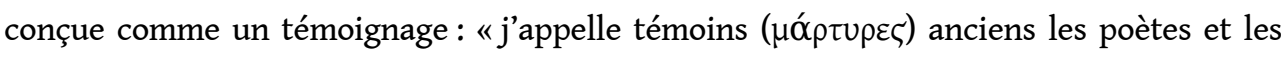
autres personnages connus de toutes sortes dont les opinions sont d'une application

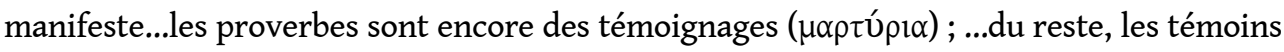
les plus accrédités sont les témoins anciens car ils sont incorruptibles »(Rhet. 1376a2-15). La formule, qui dans les exemples donnés par Aristote consiste souvent en un vers d'un 
écrivain célèbre (Rhet. 2.21, 1394a), est le point de départ des enthymèmes ${ }^{8}$. La force des sentences ${ }^{9}$, dit Aristote, «tient uniquement à la vanité des auditeurs ; en effet, ceux-ci se complaisent à voir l'orateur, en énonçant une généralité, rencontrer telle opinion qu'ils ont, eux, sur un point particulier» (Rhet. 1395b1). S'il ne développe pas la puissance tactique de la citation, en général, qui s'accroît naturellement dans la culture des Héritiers, Aristote, lui, ne confond pas la citation avec un « ornement formel » (BOMPAIRE 1958 : 379).

9 Hormis les deux passages signalés, qui visent plutôt les maximes morales et les affirmations générales (comme le montrent les exemples), le cas des citations n'est pas abordé, et les exemples où le paradeigma englobe une citation sont exceptionnels chez les rhétoriciens ${ }^{10}$. Parmi les «preuves communes » n'existe que l'exemple (avec ses « deux espèces : l'une consiste à relater des faits accomplis antérieurement; dans l'autre, on produit l'exemple lui-même : cette dernière est tantôt une parabole, tantôt un récit, comme les récits ésopiques ou les récits libyques ", Rh. 2.20,1393a).

\subsection{Constitution de florilèges}

10 C'est dans la pédagogie que la citation est essentielle ${ }^{11}$, mais sa place fondamentale est tellement évidente qu'elle passe sous l'analyse, et l'on ne donne pas de mode d'emploi des citations. Plutarque, pour sa part, recommande le butinage des jolies expressions et des fleurs de printemps (Comment lire les poètes, 11D). Elles doivent permettre, lors de l'acquisition, d'assimiler des idées majeures, et lors du réemploi de ces formules, d'apporter au discours une valeur culturelle (que l'on dirait 'ajoutée' si elle n'était pas, en quelque sorte, indispensable en tout texte), un impact qui peut compenser des failles de l'argumentation, aucun genre ne boudant ce recours. L'éducation consiste même, en bonne partie, et Plutarque reconnaît cet objectif, à donner une provision de beaux vers. Householder (1941) a montré que la liste des auteurs scolaires et celle des auteurs cités par quatorze écrivains de l'époque impériale coïncidaient exactement. A chaque génération, à l'occasion du re-traitement du patrimoine culturel, se constituent, à partir des ensembles littéraires conservés, des anthologies génériques, thématiques ou monographiques, qui sélectionnent, réduisent, et concentrent ce qui est tenu pour le cœur de l'héritage intellectuel et littéraire.

11 A la fois cause et conséquence de l'usage intensif des citations par les auteurs ces florilèges pédagogiques ou personnels se développent, en particulier sous l'impulsion des Stoïciens, sous la forme multiple de recueils d'exempla, anecdotes historiques, sentences et adages, fables, oracles, et même de comparaisons. Cette pratique culturelle quasi instinctive, qui participe de l'écriture comme récriture, est si naturelle et spontanée qu'elle peut devenir transparente à ceux qui la mettent en œuvre, comme Clément d'Alexandrie dont Van den Hoek (1996 : 236-237) dit, avec étonnement : " Il est difficile d'imaginer comment un auteur tel que Clément d'Alexandrie a pu travailler sans avoir conscience de l'interaction entre mémoire, sources intermédiaires, consultation directe des sources, et notes de lecture... ». Apollodore d'Athènes, dans son recueil de dogmes, écrit à propos de Chrysippe que "si l'on enlevait des livres de Chrysippe ce qu'il a cité d'autres auteurs, il ne resterait que des pages blanches » (Diogène-Laërce 7.181). Outre les

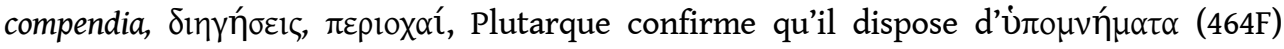
personnels, recueils de citations et de notes plus ou moins articulées, prises au fil de ses lectures. Ce travail préparatoire est même recommandé : «Tout écrivain, écrit Lucien ${ }^{12}$, 
doit prendre des notes (i.e. relever des citations), pour écrire un livre " (VAN DEN HOEK 1996 : 226). Souvent au prétexte de servir au recueillement futur de leur vieillesse, la plupart des lettrés et philosophes dut se constituer cette bibliothèque idéale de poche ${ }^{13}$, et de nombreux témoignages, tant grecs que latins, permettent de mesurer l'ampleur du phénomène littéraire et de saisir dans le détail les modalités de la composition de ces hypomnemata et le déroulement des opérations ${ }^{14}$.

\subsection{Les collections de Plutarque}

Plutarque peut donc compter, pour conforter sa mémoire et guider son travail préparatoire d'écriture, sur des collections personnelles. Il a sans doute pris assez tôt l'habitude de constituer des fiches sur ses principales lectures : 24 titres, dans le catalogue de Lamprias, relèvent directement du genre compilatoire : Actes illustres des cités, Stromates historiques et poétiques, Apophtegmes lacédémoniens, Morceaux choisis des philosophes, Livres de proverbes, Sommaire physique des opinions des philosophes, Sur les proverbes qu'on trouve chez les Alexandrins, Recueil d'oracles, etc. Dans le dispositif des collections la matrice est thématique et personnelle, et les citations, rarement très longues, sont des balises qui tracent comme un premier chemin. Le philosophe confirme lui-même cette pratique des notes préparatoires au début du traité De la tranquillité de l'âme: «Je n'ai pu m'occuper à loisir de ce que vous me demandiez; mais je vous envoie quelques réflexions générales sur la tranquillité de l'âme, qui ne sont que des notes (ن் rassemblées sur cette matière pour mon propre usage » (464F). Plutarque se révèle dans son œuvre un incontinent de la citation et l'Eroticos logos, sur lequel va se concentrer notre étude, est à ce titre suffisamment exemplaire pour que J. Irigouin et $R$. Flacelière le signalent dans leur introduction générale à Plutarque dans l'édition des Belles Lettres (p. LIX), comme l'illustration de la méthode de cet auteur.

13 On dénombre dans la liste d'Helmbold-O'Neil environ 6.840 occurrences de citations, 495 noms de sources, dont 31 figurent dans la liste d'auteurs donnée par Flacelière dans son édition de l'Eroticos, sans compter les tragiques et comiques anonymes. Le nombre indiqué de citations, en raison de l'imprécision de la catégorie et de la discrétion de certaines occurrences, ne peut avoir qu'une valeur grossière pour l'œuvre conservée. Infatigable lecteur, Plutarque a, très académiquement, confié à sa mémoire une moisson prodigieuse d'images et de phrases empruntées, et quand il dit qu'une idée ou un passage ne se trouve pas dans l'œuvre de Platon (1115C-D), on peut toujours chercher... La consultation directe des œuvres est, d'ailleurs, certaine pour de nombreux auteurs, que Plutarque, au cours de ses voyages, a eu l'occasion de lire, dans des bibliothèques (Herschbell 1992 : 3341-2). Néanmoins cette effusion prodigieuse ne s'explique pas seulement par la mémoire et la familiarité passionnelle du Béotien avec les écrivains, et devant la somme de citations on ne peut douter qu'il ait eu, lui aussi, recours à des ouvrages de seconde main, au moins dans la composition de ses ouvrages. Cette hypothèse repose également sur le constat que les textes présentent parfois des citations en séries constituées, déjà successives dans le texte source, ou articulées dans des textes secondaires. Outre ses compilations personnelles (MARTIN 1969a) le philosophe recourut donc à des compendia existants : « das anekdotische und Zitaten-Material hat P. zum grössten Teil aus Sammlungen entnommen, deren es teils für rhetorische, teils für popular-philosophische Zwecke seit langem gab» (ZIEGLER 1941 : 914). L'usage d'une gnomologie stoïcienne par Plutarque est admise (HORNA 1935, BABUT 1969, FLACELIÈRE 1997, ...), ainsi que des compilations du genre 


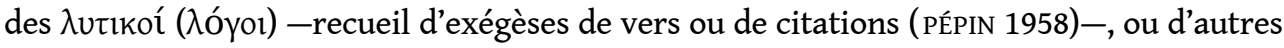
sources de seconde main (ELTER 1893) ${ }^{15}$.

Cependant, la citation, lors de l'écriture, résulte essentiellement, dans son actualisation antique, d'un acte de mémoire et non de consultation: non seulement elle est "suscitée " par la mémoire et le désir (COMPAGNON 1979), mais elle est généralement recopiée de la mémoire et non d'un texte écrit, la vérification dans l'original, une œuvre secondaire ou un répertoire d'extraits, compliquée d'ailleurs par les conditions matérielles de consultation des textes, ayant été sans doute une démarche rare ${ }^{16}$. Ce constat est particulièrement net pour Plutarque ${ }^{17}$, qui parfois reprend dans différents traités une même citation qu'il donne sous des formes différentes. Le rôle de la mémoire reste donc fondamental et l'hypothèse d'une citation « de tête » toujours valable ${ }^{18}$.

\section{Choix de citations dans l'Eroticos logos}

\subsection{Répartition et types}

Pour l'étude du dossier des citations de l'Eroticos logos on adopte de la citation cette définition provisoire et de circonstance : « reprise littérale d'une expression, d'un vers ou d'un groupe de vers, d'une phrase ou d'un membre de phrase, attribué ou non ». Dans ce texte, si le corpus reste ouvert (toutes les identification n'ont peut-être pas été faites), d'autant que le dialogue présente des lacunes parfois importantes (766D), où figuraient sûrement des citations $(763 \mathrm{~A})^{19}$, les cas litigieux représentent moins de $5 \%$ des citations retenues, car la grande majorité d'entre elles est nettement signalée et généralement poétique. En effet, sur 98 citations répertoriées (voir annexe), 11 seulement sont en prose. Les citations, souvent présentes dans d'autres traités de Plutarque ${ }^{20}$, sont de taille variable, une quinzaine des citations se limitant à un ou deux lemmes et aucune ne s'étend sur plus de 4 vers $(757 \mathrm{~A} 5,761 \mathrm{~A} 11)^{21}$, le vers pouvant être tronquée ${ }^{22}$.

Les auteurs les plus cités, tous textes confondus (recensés par Helmbold \& O'Neil) sont dans l'œuvre conservée de Plutarque : Homère (18 colonnes de 60 références en moyenne) ${ }^{23}$; Platon (14) ; Aristote $(8,5)$; Euripide (6 col. pour 366 ref.) ; Chrysippe (6,5) ; Epicure $(4,5)$; Hésiode (3,5) ; Empédocle (2,5) ; Sophocle (2,5) ; Pindare (2) ; Hérodote (2) Tragiques divers (2); Comiques divers (2); Eschyle (1); Héraclite (1); Ménandre (1) ${ }^{24}$. L'originalité de Plutarque par rapport au canon de son époque est l'importance des citations philosophiques (Aristote, Chrysippe, Epicure, Héraclite). Celles-ci ne sont pas également distribuées dans les traités, quelques textes précis (contre les Stoïciens ou les Epicuriens ; contre Hérodote...) pouvant rassembler une grande proportion des citations de l'auteur.

L'Eroticos logos est un dialogue philosophique (dont les principaux orateurs sont Daphné, Protogène, Pisias et Plutarque) rapporté à l'intérieur d'un dialogue (entre Autoboulos et Flavianos), sur le modèle platonicien du dialogue enchâssé ; il n'est pas question de se demander s'il s'agit plus d'un drame (749B) ou d'un discours (748E) puisque c'est la même chose. Il est constitué d'une suite de discours plutôt que d'entretiens, dans une forme sans doute proche de la discussion-cours pratiquée dans l'académie de Chéronée ${ }^{25}$. Les citations sont présentes pour ainsi dire à toutes les pages, sauf dans un long développement sur le mariage (753B-754D), et elles sont traditionnellement rares, voire absentes, dans les récits et les parties introductives ou conclusives. La proportion des autorités dans l'Eroticos est sensiblement différente de la moyenne, et les textes poétiques (surtout dramatiques et lyriques) y ont la part belle, les seules citations tragiques 
comptant pour près de $42 \%$ du total. Les 98 citations dénombrées se répartissent ainsi, par auteurs et catégories ${ }^{26}$ :

18 Citations épiques (19) :

- Homère (13),

- Hésiode (2),

- Dispute d'Homère et Hésiode (1),

- Autres (3)

Citations dramatiques (47):

- Euripide (13),

- Sophocle (10),

- Eschyle (7),

- Tragiques divers (12)

- Philippides (1),

- Ménandre (1),

- Comiques divers (3)

Citations lyriques (16) :

- Pindare (4),

- Solon (2),

- Sappho (2),

- Alcée (2),

- Anacréon (2),

- Archiloque (1),

- Philoxène (1),

- Mélanippides (1),

- chant (1)

- Empédocle (2),

- Xénophane (1),

- Parménide (1)

Citations philosophiques prosaïques (12) :

- Platon (6),

- Chrysippe (2),

- Héraclite (1),

- Aristippe (1),

- Ariston (1),

- Caton (1)

Comme dans les autres textes de Plutarque les citations peuvent être l'objet de remaniement, si l'on en croit nos éditions modernes des auteurs sources. Certaines résultent d'un amalgame de vers, pratique courante, déjà chez Platon ou Aristote, qui recompose un texte, parfois même en combinant des auteurs différents ${ }^{27}$. Dans l'Eroticos, on identifie clairement deux amalgames de ce genre, peut-être déjà consacrés dans des anthologies intermédiaires : en 756D11-13 se succèdent trois vers d'Euripide, provenant de deux pièces différentes; en 757E-F apparaissent trois vers de Pindare correspondant à deux fragments distincts, cités séparément dans d'autres œuvres de Plutarque ${ }^{28}$. Le texte offre également un cas de citation au second degré, la reprise d'une citation présente dans le texte : « Eros oisif » selon Euripide (757A4 et 760D5). 


\subsection{Présentation des citations}

\section{Citation anonyme ou attribuée}

24 correspond à la coutume littéraire: sauf quelques exceptions, comme Athénée qui, presque systématiquement, indique l'auteur et souvent l'œuvre qu'il cite, les écrivains taisent généralement, moins par ignorance que par coquetterie ou usage, le nom des auteurs empruntés (GANGLOFF 2006 : 110). Indiquer le nom de l'auteur, entre honnêtes lettrés, peut en effet paraître impoli pour le lecteur (VAN DEN HOEK 1996 : 229). De manière générale, la précision du nom de l'auteur est souvent inversement proportionnelle à sa célébrité ou à celle du passage mentionné, mais certaines raisons personnelles peuvent s'ajouter à ce critère ${ }^{29}$. Le nom est indiqué avant ou après la citation, le citateur se contentant parfois d'une référence globale comme en 758F1 (= Phèdre 245a), où Platon, l'auteur de la phrase anonyme, est invoqué un peu plus haut (758D7).

Le cas d'Homère est, naturellement, spécial. Il est très souvent cité dans l'œuvre de Plutarque, y compris dans l'Eroticos, mais rarement nommé ( $1 / 3$ des cas $)^{30}$; pour Platon, dont Plutarque est un lecteur intime et auquel il doit plus que des extraits isolables de son œuvre, l'attribution explicite concerne un cas sur deux (voir e.g. 758F). Ce rapport (50\% de silence $)^{31}$ est le rapport moyen pour la plupart des auteurs, philosophiques ou poétiques (Chrysippe, Tragiques, Homère,...), mais certaines exceptions devraient s'expliquer par une moindre familiarité personnelle du citateur. Ainsi Empédocle est cité plus de cent fois dans l'œuvre de Plutarque, et dans seulement trois cas son nom n'est pas donné. Si l'on écarte, comme raison potentiellement mineure, l'absence de transmission du nom de l'auteur dans les sources de Plutarque (argument faible dans le cas d'un usage d'anthologies ou de compilations, où cette précision est une règle du genre, et nul dans le cas d'une consultation directe d'un ouvrage d'auteur) la mention explicite peut s'expliquer parfois comme une forme d'auto-information, le lecteur héritant d'une précision dont l'auteur juge qu'il aurait lui-même besoin.

Cette irrégularité de la mention de l'auteur affecte aussi les extraits prosaïques, plus difficile à identifier et à délimiter, et souvent éloignés des ipsissima verba. Exceptionnellement, on constate dans les mentions une erreur d'attribution ${ }^{32}$, et l'Eroticos compte, pour sa part, une seule attribution erronée certaine, quatre vers de Sophocle attribués à Euripide: «on entend dire sur la même scène:...» (757A 4). Mais cette évaluation ne peut être que partielle, car tous les extraits n'ont pas été identifiés ${ }^{33}$.

\section{Citation intégrée ou exposée}

Le plus souvent (66\% des cas) la citation, dans l'Eroticos,est intégrée syntaxiquement à la

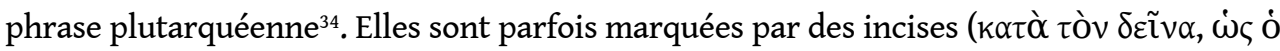
$\delta \varepsilon \tilde{I} v \alpha$ $\eta \sigma^{\prime} v$ ) mais globalement introduites de façon très variable et souple, souvent par

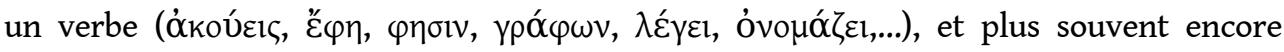
absorbées comme groupe de mots ou membre de phrase dans le texte de Plutarque ${ }^{35}$. Lorsqu'elles sont employées plusieurs fois elles sont adaptées de manière à être correctement incorporées à la phrase d'accueil ${ }^{36}$. 


\section{Citation littérale ou approchée}

28 La lettre exacte, tant du texte de Plutarque que de l'original emprunté, ne peut être absolument assurée, car on est contraint de confronter les citations de Plutarque à des éditions de textes qui résultent d'une tradition philologique longue et partielle. On constate néanmoins, surtout pour les vers, peu d'écarts dans la lettre, bien que les

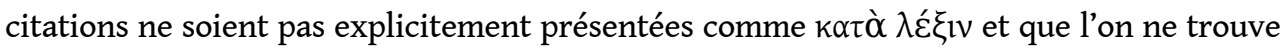
aucune des expressions consacrées telles qu' öv (explicitement), $\tilde{\omega} \delta \varepsilon \pi \omega \varsigma$ (de cette façon), etc. Quelques citations s'écartent pourtant sensiblement de l'original, comme en 760D7 où le texte de Plutarque, « $\dot{\varepsilon} v \mu \alpha \lambda \alpha \kappa \alpha \tilde{\tau} \sigma \mathrm{v} v \omega v$

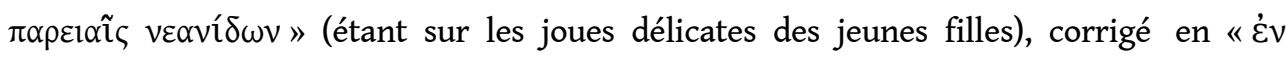

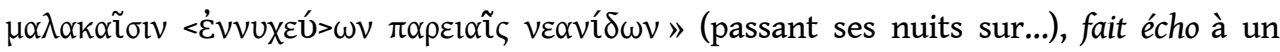

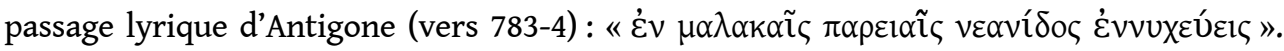
Plutarque citant souvent, comme on l'a dit, de mémoire (HELMBOLD-O'NEIL 1959 : IX), ses citations apparaissent parfois sous diverses formes dans les différents traités. C'est ainsi

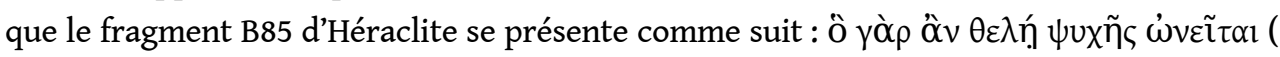

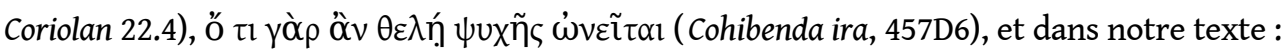

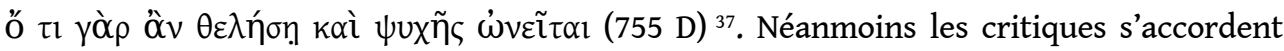
généralement à dire que « a considerable number, especially of the poetical quotations, seem to be exact and careful transcriptions » (FAIRBANKS 1897 : 77). Les paraphrases sont exceptionnelles pour les sources poétiques ${ }^{38}$, alors qu'elles sont, de manière prévisible, plus fréquentes pour les textes philosophiques en particulier platoniciens ${ }^{39}$.

\subsection{Les séquences}

Les citations ne sont pas incrustées dans le texte, et leur intégration syntaxique n'est pas un strict ajustement stylistique : elles sont constamment et continûment associées au discours comme à la pensée. On ne peut décrire simplement la dynamique intellectuelle qui engendre le texte, dans le ressac des réminiscences et le croisement des idées. Il est toutefois clair que la logique et l'organisation du texte invoqué peut se combiner avec l'ordre du discours d'accueil et l'orienter. On peut ainsi identifier des séquences qui manifestent une connexion antérieure, dans une source écrite ou la mémoire associative. Ces séquences citationnelles, généralement thématiques, sont parfois onomastiques. Deux séries de citations sont ainsi clairement repérables autour des termes $\mu$ ćp (cuisses :

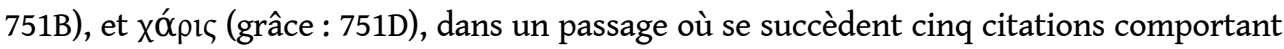
ce terme ${ }^{40}$. La suite des citations laisse apparaître aussi des groupements par auteurs, comme on en trouve naturellement chez les grands écrivains citateurs, tels Clément ${ }^{41}$. Cette liaison est parfois originale, ou bien elle est réalisée dans une œuvre intermédiaire de type compilatoire ou critique. Ainsi, en 756B7-D13, Plutarque exploite une série de cinq citations euripidéennes (sur la religion et sur Aphrodite) coupées par un extrait d'Empédocle ${ }^{42}$. Dans la suite, en 756E-F les citations suivies de deux auteurs, Parménide et Hésiode, sont déjà réunies dans le Banquet de Platon (178B) et par Aristote dans la Métaphysique (984b23-30) ${ }^{43}$, Plutarque ne retenant que les extraits et non le contexte de mention chez ces philosophes, et opérant avec les mêmes segments ordonnés son propre bricolage $e^{44}$.

30 Pour les séquences, il faut supposer soit un recours à des notes de lecture, soit un type de mémoire séquentiel, non pas de phrases seulement mais de développements ou 
d'articulation de développements; car sur ce point on ne peut vraiment mesurer la distance qu'il y a entre notre mémoire et la mémoire des Grecs -et surtout des lettrés grecs, capables de réciter d'une traite, par exemple, la Médée d'Euripide, comme le fit un jour à l'improviste Chrysippe ${ }^{45}$, et qui avaient, en permanence à l'esprit, et même, pour parodier Eschyle, "sur le bout de la langue ", des kilo-mètres de vers d'Homère, d'Hésiode, d'Archiloque, etc.

\section{Fonctions des citations}

\subsection{Fonction persuasive}

Les citations, toujours apparentes, même lorsqu'elles ne sont pas crûment et théâtralement exhibées, sont dans le texte en service commandé. Elles ne sont pas seulement le symptôme d'une écriture et la trace d'une genèse de la pensée, elles constituent -et c'est l'aspect mis en valeur en d'autres termes par la rhétorique- des outils de persuasion et de séduction. Elles sont un « argument d'autorité ", même lorsque leur auteur n'est pas nommé car, génériquement repérables par le mètre, elles témoignent, au pire, de l'insuffisance culturelle du lecteur qui n'en a pas identifié l'auteur... Plutarque n'exclut d'ailleurs aucun auteur, même ceux dont ils critiquent la doctrine générale, parfois habilement pour s'assurer l'appui de philosophes auxquels prétend s'adosser précisément l'adversaire. Epicure est du nombre ${ }^{46}$, et les poètes en général, qui sont pédagogiquement dangereux, si l'on ne fait pas dans leurs œuvres une prudente sélection et un commentaire protreptique ${ }^{47}$. De manière caractéristique, des autorités sont parfois retournées par l'adversaire, et successivement utilisées comme témoins à charge puis à décharge d'une même thèse, voire par un même orateur et dans une même intervention, comme dans la tirade où Daphné, défenseur de l'amour hétérosexuel, présente sur pièces Solon d'abord comme ridicule, puis en sage : «Comme tu as bien fait, di-il, de citer ( $\dot{\varepsilon} \mu v \eta \dot{\sigma} \sigma \eta \eta)$ Solon! On doit le prendre en effet comme type de l'amant: 'Tant qu'il aime des garçons la jeunesse en fleur, <désirant> la douceur des cuisses et des lèvres'... D'autres peuvent se moquer de ces poètes qui engagent les amants à examiner des cuisses et des hanches comme le font les sacrificateurs et les devins! Moi

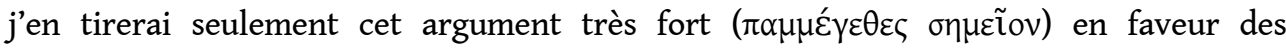
femmes... [...] Aussi, à mon avis, Solon a-t-il écrit les vers que j’ai cités quand il était encore jeune et 'tout gonflé de sperme', comme dit Platon; devenu vieux, il s'exprimait ainsi: 'Maintenant je me plais aux travaux de Cypris, de Dionysos et des Muses, qui apportent la joie aux hommes'. Ainsi Solon, au sortir des ouragans et des tempêtes de l'amour des garçons, avait établi sa vie dans un certain calme, celui du mariage et de la philosophie » $(751 \mathrm{C}-\mathrm{E})^{48}$. Le recours aux auteurs, comme à un réservoir d'assesseurs ou de témoins, autorise une circulation libre et ne condamne pas à un alignement sur l'idée introduite avec eux. Euripide, à qui on prête tant et qui, selon ses personnages, défend dans ses tragédies toutes les positions, par des sentences de circonstance, est naturellement exploité par Plutarque qui, par interlocuteurs fictifs interposés, fait flèche de tout bois, soit pour confirmer, soit pour combattre une idée ${ }^{49}$. Puisque «la poésie ne se soucie pas du tout de la vérité » (Comment...17D), il est légitime d'orienter les emprunts qu'on lui fait. 


\subsection{Fonction dynamique}

32 La citation n'est donc pas toujours une caution mais sert éventuellement de contrepoint. Au-delà de sa mission persuasive elle a, foncièrement, un rôle dynamique essentiel, car elle suscite et entraîne un développement, un commentaire ou une réfutation. Elle peut donc être formulé a contrario : «Il n'est ni oisif, comme Euripide l'en accusait, ni inapte à faire campagne, et il n'est pas vrai 'qu'il passe les nuits sur les joues délicates des jeunes filles'... ${ }^{50}$ L'autorité citée est ainsi introduite sans approbation: «Je ne sais pas ce que Ménandre avait dans l'esprit, je ne le comprends pas lorsqu'il dit : « cette maladie est une crise l'âme, celui qu'elle atteint porte une blessure interne »; en réalité, c'est le dieu qui est cause de tout» (763B). Les références s'imposent, mais parfois dans un contexte critique, pour être dépassées : « Euripide ne s'est émerveillé que du moindre des bienfaits [d'Eros] quand il a dit « ... », car l'amour...» (762B4). La citation offre donc davantage de possibilités rhétoriques et discursives que les sentences dont le seul prolongement est, selon Aristote (Rhet. 2.21, 1394b), l'ajout d'un épilogue, seulement lorsque la maxime avancée comporte un paradoxe. Dans la moitié des cas que présente l'Eroticos, en effet, la citation introduite est commentée (renforcée ou contestée), et permet une progression de la thématique. Elle est parfois aussi l'occasion d'introduire de façon incidente, tel un pivot, des idées nouvelles : ainsi un segment d'hexamètre homérique (759F) contenant le

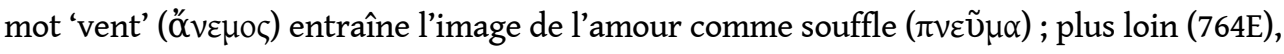
une citation d'Euripide sur l'attachement aux choses terrestres, finissant sur «par

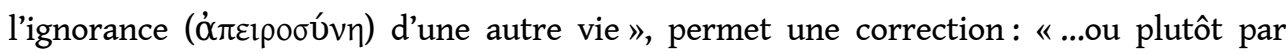

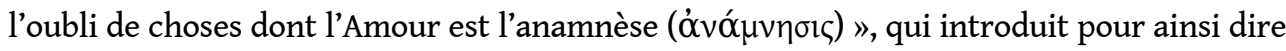
naturellement à la théorie platonicienne de la réminiscence et de la psychagogie de l'âme par l'amour ${ }^{51}$. La citation autorise, dans le développement d'une argumentation philosophique, tenue à un certain déploiement méthodique, des diversions et des réorientations. Citée comme témoin, elle, dont la légitimité et la pertinence dans le discours n'est jamais mise en cause par un interlocuteur, peut devenir l'occasion d'un nouveau départ.

\subsection{Fonction ornementale}

Sa valeur «ornementale» est à la fois évidente et ambiguë. Si le terme est entendu comme synonyme de 'décoratif' ou 'discursivement immotivé', on peut supposer que fort peu de citations, dans un texte de Plutarque, encourent cette qualification réductrice. Il y a sûrement, ça et là, un fragment de discours que Plutarque préfère dire par la voix d'un autre qu'il a assimilé, ou bien des remarques ou des détails accessoires ou adventices qui surgissent, presque automatiquement, au cours de l'écriture, sans qu'ils apportent à l'argumentation un élément original ou pertinent. C'est, sans doute, le cas de tel extrait pindarique extravagant sur les dryades $(757 \mathrm{E})^{52}$, de quelques lexèmes sophocléens amenés comme en passant (758E11) ou d'expressions homériques bien frappées qui s'invitent (761B12, 767A7...). Même si la rhétorique antique semble encourager cette voie, puisque le rôle même de la citation est, d'après Quintilien, de "donner de l'autorité à leurs plaidoyers ou de l'ornement à leur éloquence... pour marquer leur érudition et pour plaire » (Instit. orat. 1.8.10), il parait naïf de s'en tenir là̆${ }^{53}$. Il y a de multiples façons de contribuer à une argumentation, et de participer à une stratégie discursive, surtout lorsqu'on entre au service d'un philosophe rompu par son maître aux subtilités de la 
discussion efficace ${ }^{54}$. Le plaisir de l'auditoire est un objectif incontournable et la visée hédonique de la citation est incontestable parce que le plaisir suscité est un atout de persuasion.

Inutile d'invoquer, pour justifier l'abondance de citations qu'on dirait luxueuse, le projet pédagogique qu'aurait Plutarque et qui devrait, conformément à ses principes, s'accompagner, quels qu'ils soient, de sentences héritées et de mémentos poétiques, tirant partie du "potentiel mnémotechique et psychagogique » de la poésie (BRÉCHET 2005 : 112-113). Lorsqu'elle semble décalée, voire gratuite, la citation sert souvent le dialogue, à défaut de ses membres : elle permet des pauses, comme lorsque Zeuxippe en riant, cédant à sa passion "phileuripidéenne", déclame deux vers (sans destinataire présent) à l'adresse des femmes orgueilleuses (755B9); une dramatisation utile, comme lorsque le père du narrateur fait son entrée dans le débat en marquant en ces termes son hostilité à l'orateur précédent : «Ces mots, au peuple argien, feront prendre les armes [ trag. adesp.]» (752C); ou une évolution discrète, comme dans ce passage du texte consacré à Arès et Hadès (760D-762A), qui semble s'égarer dans des trivialités sur Hadès (il est inflexible et implacable) mais permet à Plutarque, par les reprises et gloses des citations, de transformer une mention qui pouvait être mécanique ou réflexe en un développement pertinent progressant par légers déplacements : Arès-le courage-le courage devant la mort-le triomphe de la mort-la résurrection-la résurrection symbolique-la révélation amoureuse ; Plutarque conclut en évoquant le retour des amants vers la lumière du jour, qui préfigure le développement suivant sur la révélation amoureuse qui fait passer du royaume d'Hadès au champ de la vérité $(765 \mathrm{~A})^{55}$. La citation peut, certes, être compulsive et, le cas échéant, maladroite, un faux-pas dans le parcours de l'auteur rhapsodiant, mais il faut se garder d'en chercher la justification dans sa seule valeur sémantique immédiate. Elle est un facteur déterminant dans les processus réflexifs et créatifs qu'elle active chez l'écrivain, et dans les processus psychologiques qu'elle conditionne chez le lecteur.

\subsection{Les subversions}

L'intégration syntaxique et l'intégration argumentative témoignent d'une adoption complète de la citation, dans le tissu du texte, et s'accompagne aussi d'une véritable manipulation. Celle-ci est facilitée par la décontextualisation des citations. L'isolement, qui vaut de manière générale pour la pratique citationnelle, est profitable, puisque l'élusion du contexte est un gage de rendement pour une sentence. Prélevée seule, la citation, parfois très brève, gagne en plasticité et sert au-delà de son usage premier dans le texte source. Le décalage induit, qui peut aller de l'extension au dévoiement total et au « contresens » (au regard naturellement d'une interprétation de la citation dans le texte originel), est parfois involontaire ${ }^{56}$. Le recours signalé plus haut à des textes de médiations et à des collections empêche d'incriminer formellement l'auteur de ce détournement. On a pu constater ainsi que Clément d'Alexandrie est totalement ignorant du contexte des citations qu'il donne (FAIRBANKS 1969) ${ }^{57}$. Le commerce des citations est objectivement libéré par le fait aussi que, souvent, les énonciateurs sont omis dans les ouvrages de seconde main qui mettent sur le compte d'un auteur tout ce qu'il rapporte, ignorant les énonciateurs intégrés sur le mode : 'Aristote dit que [X prétend à tort que $\mathrm{Y}$ disait que] phrase $a^{58}$. Ce contexte premier nous manque également souvent (en particulier dans les nombreux fragments), pour déterminer précisément ces dérives. 
Mais le code d'usage des citations, comme on a pu déjà le percevoir dans l'absence de formalisation théorique et comme le montre la grande richesse et diversité des pratiques exégétiques, est dans l'antiquité beaucoup plus souple. Non astreints à une déontologie "moderne », l'auteur et le lecteur, d'accord sur ce point, se soucient modérément de «l'intention de l'auteur » d'une citation qu'ils recrutent: celle-ci n'est pas indifférente, mais l'exégèse se doit de viser plus loin, et l'on tend moins, dans l'échange de paroles, à établir ce qu'une autorité a voulu dire que ce qu'elle peut (éventuellement à son insu) exprimer -et ce que son énoncé peut permettre à d'autres d'exprimer. Il convient de chercher la raison ( $\alpha i \tau i ́ \alpha)$ des paroles poétiques, et celle-ci ne peut être valable que si, aux yeux du lecteur, "elle est conforme au bien» (comment... 28B). Après la censure platonicienne par excision, Plutarque recommande, dans Comment lire les poètes, une censure par falsification, lorsque le texte admis comporte une indécence : il s'agit de modifier la lettre quand elle pêche, Plutarque proposant quelques exemples de redressement moral de citations vicieuses. C'est ainsi que les « corrections allant contre le texte » ( $\pi \alpha \rho \alpha \delta$ เор$\theta \omega ́ \sigma \varepsilon ı \varsigma)$ préconisées par les Stoïciens (33B9), deviennent de simples

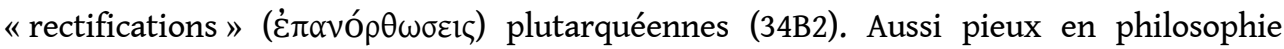
qu'Augustin en théologie, lequel admettait toutes les interprétations de l'Ecriture, même fausses, pourvu qu'elles soient conformes à la charité (Doct. christ. 1.36.40), Plutarque tolère tous les maquillages pourvu qu'ils encouragent la vertu ${ }^{59}$.

Le citateur emprunte des mots, une autorité, -mais le sens reste négociable. De ces sentences, gravées dans l'espace de parole par les poètes et les penseurs, et qui ne peuvent être réfutées que par d'autres sentences (BRÉCHET $2005: 110$ ), il est admis par principe qu'elles sont toujours à leur place. Comme le pouvoir, parce que c'est un pouvoir, on pourrait presque dire qu'il n'y a pas d'usage de la citation mais seulement des abus de la parole rapportée.

Le détournement d'autorité est donc monnaie courante dans l'économie citationnelle ${ }^{60}$, et les exemples d'exégèses biaisées ou d'interprétations déloyales abondent dans les traités de Plutarque ${ }^{61}$, et dans l'Eroticos en particulier. Lorsqu'il cite Pindare en 751D, Plutarque amalgame une tradition hésiodique et une formule pindarique: "Ainsi Pindare dit

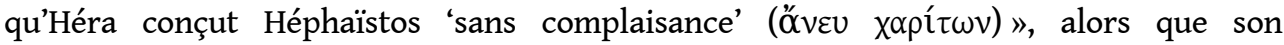
compatriote emploie l'expression (Pyth. 2.42) pour le fantôme d'Héra dans les bras d'Ixion, le père des Centaures ${ }^{62}$. L'extension de la portée des citations à des réalités proches (selon le principe stoïcien de "transfert et déplacement de sentences utiles sur des domaines similaires $»)^{63}$ permet de faire valoir pour le désir sans amour un énoncé qui concerne initialement la mort (756E6), ou de suggérer dans un indéfini un autre signifié que celui visé par le texte original $(756 \mathrm{~B})^{64}$. Dans ce bricolage citationnel le sens de la citation dans le texte d'accueil peut, par omission ou distorsion, aller à l'encontre du sens d'origine ${ }^{65}$, faisant passer pour négatifs des propos sur Aphrodite, qui ne le sont pas (757A7), ou un vers d'amour de Sappho sur une adolescente pour un éloge des relations conjugales $(758 \mathrm{D} 8)^{66}$.

\section{Conclusion}

Prince de l'entreglose, comme dirait Montaigne -qui en fut un autre-, Plutarque sait que citer n'est pas jouer, ou paraître, mais écrire. Dans l'Eroticos logos, œuvre "dramatique " exceptionnellement riche en citations, puisque le traité didactique Comment lire les poètes compte juste le double de références pour une longueur équivalente ${ }^{67}$, apparaît 
nettement la portée réelle de l'usage des citations, qui tient moins dans l'acte citationnel lui-même que dans ce dont elles sont le symptôme mental: la circulation constante de l'écrivain dans un univers culturel balisé, où les textes se construisent ouvertement dans la polyphonie avec des morceaux de déjà-là. L'absence de « droit d'auteur » (ZIEGLER 1949, STEMPLINGER 1912), d'exigence littéraliste, de code exégétique et de déférence obligée envers les Anciens, tout vénérables soient-ils -ces contraintes étant abandonnées aux grammairiens-, joue un rôle décisif et profondément stimulant dans la création intellectuelle antique et la dynamique interne des œuvres, y compris lorsqu'elles ont mais n'est-ce pas le régime commun de la production graphique antique ?- une forte ambition littéraire.

Les broderies plutarquéennes, nourries d'associations d'idées, d'images rémanentes et de sentences toutes faites, qui offrent une version savante et rénovée de la rhapsodie archaïque, et un prolongement sur le mode oral et écrit de la com-position poétique, défient les interprétations modernes qui, acquises à la grandeur positive de l'intertextualité, peinent cependant à traiter organiquement le texte lorsqu'ils y décèlent des traces ou des empreintes venues d'ailleurs. Rien qui suggère dans la pratique ancienne de l'écriture associée la représentation que donne un sémioticien moderne et classique du statut de la citation : « la citation est un corps étranger dans mon texte, parce qu'elle ne m'appartient pas en propre, parce que je me l'approprie » (COMPAGNON $1979: 31$ ).

Sans théorie rhétorique ni poétique de la citation, Plutarque, à l'image de ses contemporains et dans le reflet de sa culture, use d'une pratique universelle, qui est fondue dans l'idée même de création, et se présente comme un réflexe conditionné de la pensée comme de l'écriture grecque.

\section{Annexe}

\section{Liste des citations de Plutarque dans l'Eroticos logos}

La liste est organisée par catégories génériques ${ }^{68}$. Le premier numéro indique la place relative de la citation dans le dialogue, le second la numérotation conventionnelle dans l'œuvre de Plutarque (R. Estienne); puis sont précisés pour chaque citation si elle est anonyme (An) ou accompagnée du nom de l'auteur (Nom), syntaxiquement intégrée (I) ou non (NI) ${ }^{69}$, éventuellement décontextualisée (Déc) ou fortement contextualiséz (Cont) ; la référence du passage cité, la longueur en mots (M), en lignes de l'édition Belles Lettres (L), ou en vers (V), les mots introducteurs, et d'éventuelles remarques.

\begin{tabular}{|l|l|l|l|l|l|l|}
\hline$n^{\circ}$ & ref. & Nommé & Intégré & Contextualisé & source & Mot, Ligne, Vers \\
\hline
\end{tabular}

\section{Homère (14)}

\begin{tabular}{|c|c|c|c|c|c|}
\hline 6 & 751A1 & Nom & I & Homère Iliade, 21.252 \& 24.315 & $2 \mathrm{M}$ \\
\hline
\end{tabular}




\begin{tabular}{|c|c|c|c|c|c|c|c|}
\hline 36 & 757B3 & Nom & NI & & \multicolumn{2}{|l|}{ Homère Il., 5.31 \& 83} & $2 \mathrm{M}$ \\
\hline \multicolumn{8}{|c|}{ Homère appelle ce dieu « ... » et « ... » } \\
\hline 40 & 757E5 & Nom & I & & \multicolumn{2}{|l|}{ Homère Odyssée, 5.68} & $1 \mathrm{M}$ \\
\hline \multicolumn{8}{|c|}{...ni cette vigne qu’Homère dit « cultivée » } \\
\hline 44 & $758 \mathrm{C} 1$ & An & I & Déc & \multicolumn{2}{|l|}{ [Homère, Od., 5.372] } & $2 \mathrm{M}$ \\
\hline \multicolumn{8}{|c|}{ ce n'est pas « sans le secours d'un dieu » qu'elles atteignent... } \\
\hline 50 & 759A9 & An & I & & \multicolumn{2}{|l|}{ [Homère Il., 7.121] } & $1,5 \mathrm{~V}$ \\
\hline \multicolumn{8}{|c|}{ se dépouille de sa fureur guerrière « tandis que ses valets... } \\
\hline 55 & 759F3 & An & I & & \multicolumn{2}{|l|}{ [Homère Il., 17.57] } & $0,5 \mathrm{~V}$ \\
\hline \multicolumn{8}{|c|}{ « Mais que, soudain, le vent se lève » et apporte... } \\
\hline 60 & 761B10 & Nom & I & & \multicolumn{2}{|l|}{ Homère Il., 2, 362} & $4 \mathrm{M}$ \\
\hline \multicolumn{8}{|c|}{ parce que ce poète répartissait les Achéens " par tribus et par clans" } \\
\hline 61 & 761B13 & An & NI & & \multicolumn{2}{|l|}{ [Homère Il., 13.131 \& 16.215] } & $1 \mathrm{~V}$ \\
\hline \multicolumn{6}{|c|}{ pour que ce soit : « le bouclier touche... } & \multicolumn{2}{|l|}{ H. cité juste avant } \\
\hline 64 & $761 \mathrm{~F} 6$ & An & I & Cont & [Homère , Il., 9.158] & & $2 \mathrm{M}$ \\
\hline \multicolumn{6}{|c|}{...et cesse pour eux seuls d'être « inflexible, implacable »... } & \multicolumn{2}{|l|}{ ordre inversé } \\
\hline 67 & $762 \mathrm{E} 4$ & An & NI & Cont & [Homère , Od., 19.40] & & $0,5 \mathrm{~V}$ \\
\hline \multicolumn{6}{|c|}{ dire comme Télémaque : «...» } & \multicolumn{2}{|l|}{ Télémaque nommé } \\
\hline 78 & $764 \mathrm{~A} 6$ & An & I & & [Homère , Il., 12.453] & & $2 \mathrm{M}$ \\
\hline \multicolumn{8}{|c|}{ en ce qui concerne « les propos... } \\
\hline 86 & 767A7 & An & I & & [Homère , Il., 23.29] & & $0,5 \mathrm{~V}$ \\
\hline \multicolumn{8}{|c|}{ estime autant que les qualités du cheval Podarge celles « d'Aitha... } \\
\hline 97 & 770A5 & An & I & & [Homère, Od., 6.183-184] & & $1,5 \mathrm{~V}$ \\
\hline
\end{tabular}


Hésiode (2)

\begin{tabular}{|c|c|c|c|c|c|c|c|}
\hline 20 & $753 \mathrm{~A} 6$ & Nom & NI & Cont & Hésiode, T.J., 696-698 & & $3 \mathrm{~V}$ \\
\hline \multicolumn{8}{|c|}{$\begin{array}{l}\text { commettre une absurdité ridicule en prenant le contre-pied d'Hésiode, si, } \\
\text { alors qu'il dit « ... », nous allions... }\end{array}$} \\
\hline 32 & 756F2 & Nom & | & Cont & Hésiode, Théogonie, 120 & & - \\
\hline \multicolumn{6}{|c|}{ Hésiode témoigne d'une science plus profonde quand il fait de l'amour... } & $\begin{array}{l}\text { Paraphrase } \\
\text { Abus }\end{array}$ & \\
\hline
\end{tabular}

\section{Hexamètres épiques (4)}

\begin{tabular}{|c|c|c|c|c|c|}
\hline 38 & 757D8 & An & $\mathrm{I}$ & ¿Callimaque? & $1 \mathrm{~V}$ \\
\hline \multicolumn{6}{|c|}{ ils invoquent Aristée « qui le premier... } \\
\hline 62 & $761 \mathrm{E} 7$ & An & $\mathrm{I}$ & ¿Callimaque? & $1 \mathrm{~V}$ \\
\hline \multicolumn{6}{|c|}{ qui, d'après la fable, " pour une grande année... } \\
\hline 81 & $764 \mathrm{~F} 7$ & An & $\mathrm{I}$ & ¿Callimaque? & $1 \mathrm{~V}$ \\
\hline \multicolumn{6}{|c|}{ «Sur elle... », et elle s'imagine... } \\
\hline 66 & 762D6 & An & $\mathrm{I}$ & [Tournoi d'Homère et d'Hésiode 274] & $1 \mathrm{~V}$ \\
\hline
\end{tabular}

\section{Empédocle, Xénophane, Parménide, Héraclite (5)}

\begin{tabular}{|c|c|c|c|c|c|c|c|}
\hline 26 & 756D2 & Nom & NI & & \multicolumn{2}{|c|}{ Empédocle, frg B 17, 20} & $2 \mathrm{~V}$ \\
\hline \multicolumn{6}{|c|}{ Lorsque mon cher tu lis chez Empédocle «... } & \multicolumn{2}{|c|}{ Abus (cs). Commenté } \\
\hline 28 & 756E1 & Nom & NI & & \multicolumn{2}{|l|}{ Empédocle, frg B 151} & $1 \mathrm{M}$ \\
\hline \multicolumn{6}{|c|}{ Empédocle la nomme «... } & & \\
\hline 75 & 763D1 & Nom & I & Cont & \multicolumn{2}{|l|}{ Xénophane, frg B 13} & $1 \mathrm{~L}$ \\
\hline \multicolumn{6}{|c|}{ comme Xénophane ordonna aux Egyptiens de « ne pas... } & \multicolumn{2}{|l|}{ Style ind. } \\
\hline 31 & 756F1 & Nom & NI & Cont & Xénophane, frg B 13 & & $1 \mathrm{~V}$ \\
\hline \multicolumn{6}{|c|}{ il écrit dans sa Cosmogonie « ... } & \multicolumn{2}{|l|}{ Nom d'œuvre } \\
\hline
\end{tabular}




\begin{tabular}{|l|l|l|l|l|l|l|}
\hline 22 & $755 \mathrm{D} 8$ & Nom & I & \multicolumn{1}{|c|}{ Héraclite, frg B 85 } & $2 \mathrm{~V}$ \\
\hline
\end{tabular}

\section{Euripide (13)}

\begin{tabular}{|c|c|c|c|c|c|c|c|}
\hline 21 & 755B9 & Nom & $\mathrm{NI}$ & & \multicolumn{2}{|l|}{ Euripide, Nauck ${ }^{2}$ p. 678} & $1 \mathrm{~V}$ \\
\hline \multicolumn{8}{|c|}{ Comme il était féru d'Euripide, il dit « ... } \\
\hline 23 & $756 \mathrm{~B} 7$ & An & I & Cont & \multicolumn{2}{|l|}{ [Euripide, Bacchantes, 203] } & $1 \mathrm{~V}$ \\
\hline \multicolumn{8}{|c|}{ on ne pourrait pas trouver « même si... } \\
\hline 24 & 756B14 & Nom & $\mathrm{NI}$ & & \multicolumn{2}{|l|}{ Euripide, Mélanippe, 1} & $1 \mathrm{~V}$ \\
\hline \multicolumn{6}{|c|}{$\begin{array}{l}\text { quel tumulte Euripide provoqua pour avoir commencé sa Mélanippe } \\
\text { par ce vers : «... }\end{array}$} & \multicolumn{2}{|l|}{ Nom d'œuvre } \\
\hline 25 & $756 C 3$ & Nom & $\mathrm{NI}$ & & \multicolumn{2}{|l|}{ Euripide, Mélanippe, 1} & $1 \mathrm{~V}$ \\
\hline \multicolumn{8}{|c|}{$\begin{array}{l}\text { il remplaça le vers en question par celui que nous lisons aujourd'hui : } \\
« . .\end{array}$} \\
\hline 33 & 757A4 & An & NI & & \multicolumn{2}{|c|}{ [Euripide, Danaé, Nauck ${ }^{2}$ p. 455] } & $1 \mathrm{~V}$ \\
\hline \multicolumn{6}{|c|}{ on entend dire sur la même scène : «... } & \multicolumn{2}{|l|}{$\begin{array}{l}\text { Incongru } \\
\text { Repris en 760D5 }\end{array}$} \\
\hline 43 & 758B14 & An & I & Déc & [Euripide, Bacchantes, 66] & & $1 \mathrm{~V}$ \\
\hline \multicolumn{6}{|c|}{ la complaisance qui donne lieu, en vérité, à « ... } & \multicolumn{2}{|l|}{$\begin{array}{l}\text { Incongru } \\
\text { Repris en 760D5 }\end{array}$} \\
\hline 51 & $759 \mathrm{~B} 8$ & An & I & & [Euripide, Hippolyte, 478] & & $2 \mathrm{M}$ \\
\hline \multicolumn{6}{|c|}{ ni œuvre de l'art, ni «...» } & \multicolumn{2}{|l|}{ Quasi littérale } \\
\hline 65 & $762 \mathrm{~B} 7$ & Nom & $\mathrm{NI}$ & & \multicolumn{2}{|c|}{ Euripide, frg.663 Nauck ${ }^{2}$ p. 569} & $1,5 \mathrm{~V}$ \\
\hline \multicolumn{8}{|c|}{ disant $« \ldots$} \\
\hline 77 & $763 \mathrm{~F} 4$ & Nom & I & & \multicolumn{2}{|c|}{ Euripide, frg. 595 Nauck $^{2}$ p. 549} & $1 \mathrm{~V}$ \\
\hline \multicolumn{8}{|c|}{ l'union de ces couples n'est pas telle qu'Euripide dit «... } \\
\hline 80 & 764 E 5 & Nom & I & & Euripide, Hippolyte 193-196 & & $3 \mathrm{~V}$ \\
\hline
\end{tabular}




\begin{tabular}{|c|c|c|c|c|c|c|}
\hline \multicolumn{5}{|c|}{ «Aussi... » comme dit E., « à cause de... », ou plutôt de... } & \multicolumn{2}{|c|}{ Corrigé } \\
\hline 80 & $766 \mathrm{C} 3$ & Nom & I & \multicolumn{2}{|c|}{ Euripide, Hippolyte 7} & $1 \mathrm{~V}$ \\
\hline \multicolumn{7}{|c|}{ l'amour, comme les autres dieux, « se plaît », comme dit E. à... } \\
\hline 92 & 767F5 & An & $\mathrm{I}$ & \multicolumn{2}{|c|}{ [Euripide, frg.1084 Nauck ${ }^{2}$ p.703] } & $1 \mathrm{~V}$ \\
\hline \multicolumn{7}{|c|}{ alors, « abandonnant ... » elle quitta } \\
\hline 98 & 770A10 & An & I & \multicolumn{2}{|c|}{ [Euripide, frg.898 Nauck $^{2}$ p.648] } & $0,5 \mathrm{~V}$ \\
\hline Ain & poè & ent & & que le ciel... & & \\
\hline
\end{tabular}

\section{Sophocle (10)}

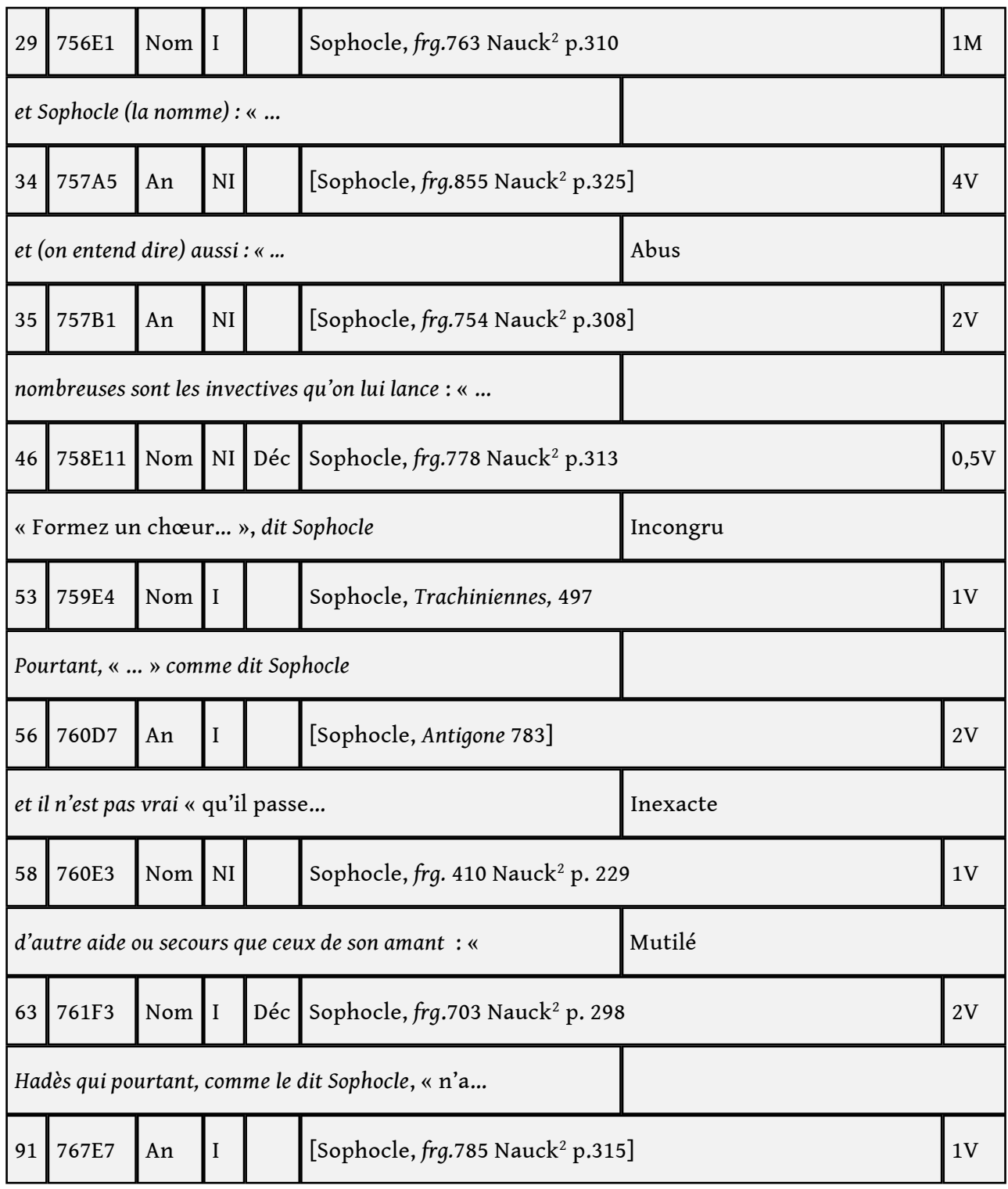


La fidélité mutuelle... « est l'œuvre...

\begin{tabular}{l|l|l|l|l|l}
94 & $768 \mathrm{E} 10$ & Nom & I & & Sophocle, frg.779 Nauck $^{2}$ p.313
\end{tabular}

et vraiment, comme le dit Sophocle « de tels amis...

Eschyle (7)

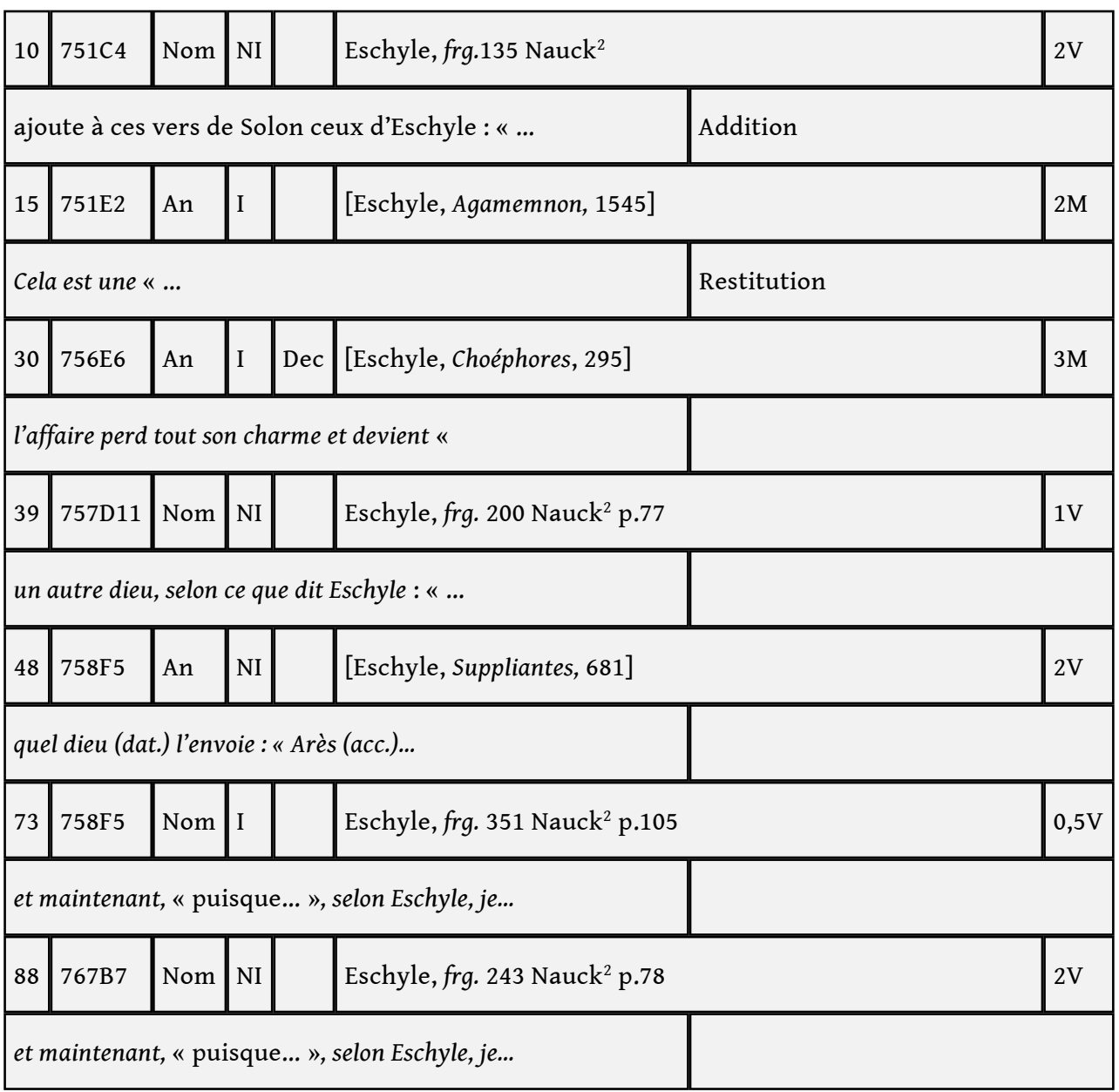

Poètes Tragiques (12)

\begin{tabular}{|c|c|c|c|c|c|c|}
\hline 1 & 750A11 & An & I & \multicolumn{2}{|c|}{ [Trag. Nauck $^{2}$, p.632] ¿Euripide? } & $1 \mathrm{~V}$ \\
\hline \multicolumn{6}{|c|}{...lui dont $[. .$.$] « l'oubli...$} & \\
\hline 4 & $750 \mathrm{E} 8$ & An & I & \multicolumn{2}{|c|}{ [Trag. Nauck ${ }^{2}$, p.916, Adesp. 401] } & $2 \mathrm{~V}$ \\
\hline \multicolumn{5}{|c|}{ Tu connais cette réplique d'un personnage $[. .]:$. «... } & \multicolumn{2}{|c|}{ Introduit un parallèle } \\
\hline 69 & 762E11 & An & I & \multicolumn{2}{|c|}{ [Phrynichos, frg.17 Nauck ${ }^{2}$ p.724] } & $1 \mathrm{~V}$ \\
\hline
\end{tabular}




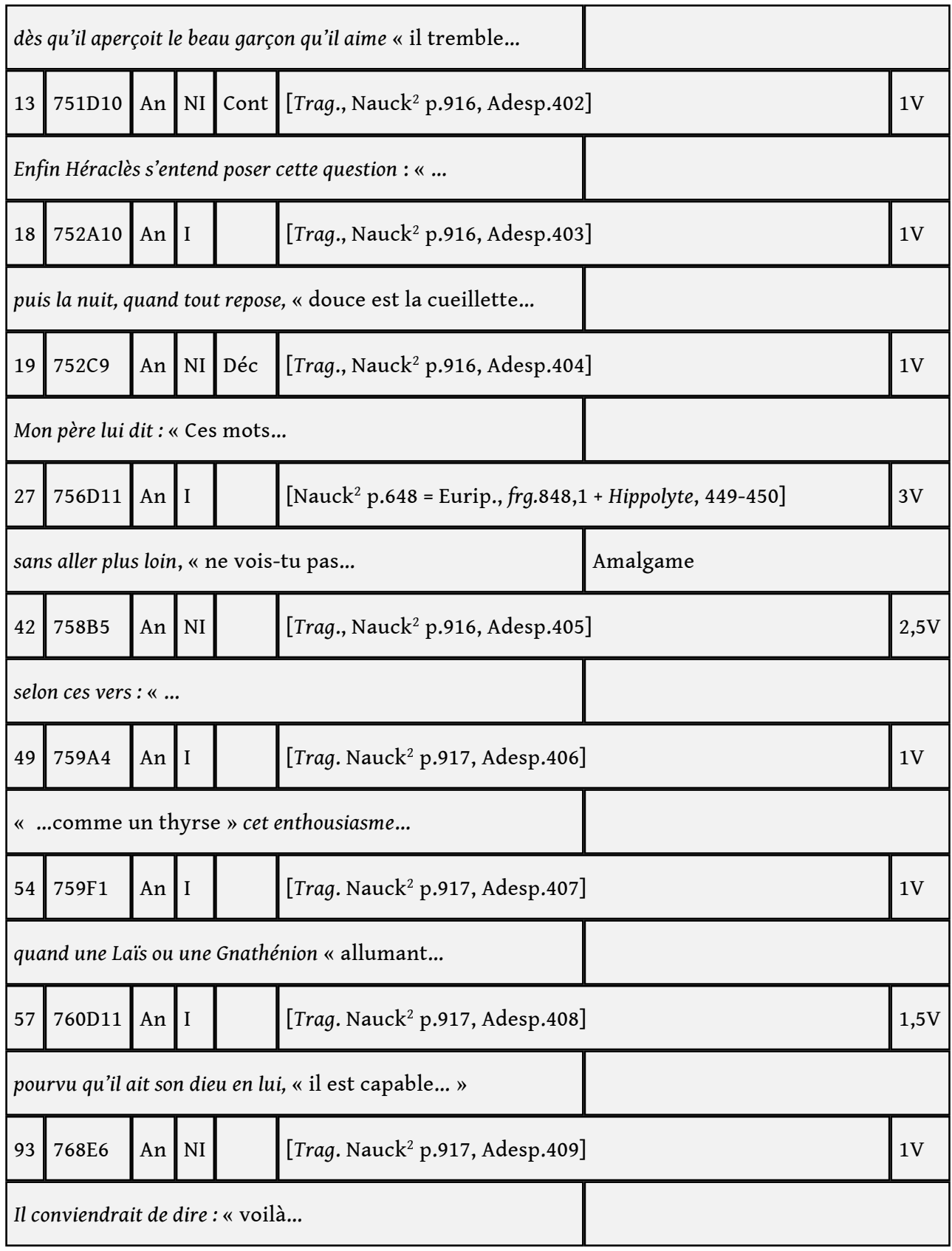

\section{Poètes Comiques (5)}

\begin{tabular}{|l|l|l|l|l|l|l|l|}
\hline 5 & $750 \mathrm{~F} 3$ & Nom & NI & Cont & \multicolumn{2}{|l|}{ Philippidès frg.31 (Kock, 3.310) } & $1 \mathrm{~V}$ \\
\hline \multicolumn{7}{|l|}{ Comme Philippidès l'auteur comique [...] : « ... } & \multicolumn{2}{l|}{ comparaison } \\
\hline 72 & $763 \mathrm{~B} 7$ & Nom & NI & & Ménandre, frg.568 (Körte, p.185) & $1,5 \mathrm{~V}$ \\
\hline
\end{tabular}




\begin{tabular}{|c|c|c|c|c|c|c|}
\hline \multicolumn{5}{|c|}{$\begin{array}{l}\text { "l'homme qui }[. . .] \text { se voit demander si « il préfère... » et répond « les } \\
\text { deux... » ne semble pas... }\end{array}$} & \multicolumn{2}{|c|}{$\begin{array}{l}\text { Trag ? ou Com ? } \\
\text { Vers disjoints }\end{array}$} \\
\hline 95 & 769B6 & An & NI & \multicolumn{2}{|c|}{ [Com., Kock 3.450, Adesp.222-4] } & $3 \mathrm{~V}$ \\
\hline & & & & & \multicolumn{2}{|l|}{ Isolé } \\
\hline 96 & 769D10 & An & NI & \multicolumn{2}{|c|}{ [Com., Kock 3.450, Adesp.221] } & $1 \mathrm{~V}$ \\
\hline \multicolumn{5}{|c|}{ dire, comme dans la comédie : « ... } & \multicolumn{2}{|c|}{ Comédie } \\
\hline
\end{tabular}

\section{Pindare (4)}

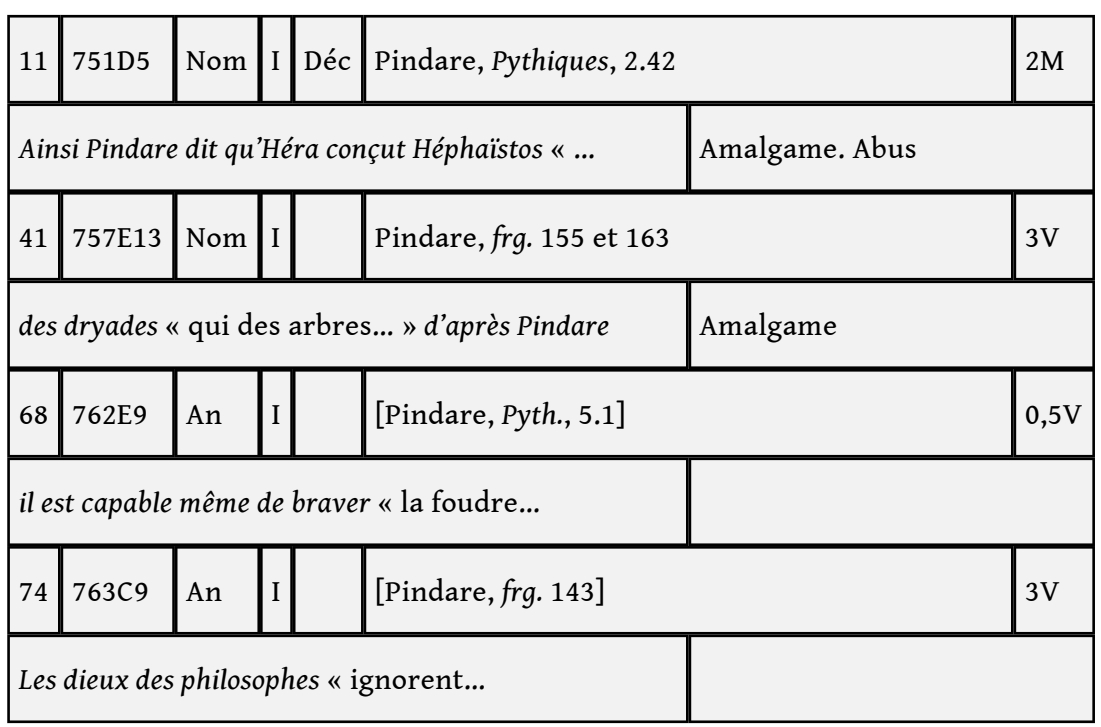

\section{Autres poètes lyriques (12)}

\begin{tabular}{|c|c|c|c|c|c|c|c|}
\hline 2 & $750 \mathrm{~B} 3$ & An & I & & \multicolumn{2}{|l|}{ [Archiloque, frg.170 (Lasserre)] } & $1 \mathrm{~V}$ \\
\hline \multicolumn{6}{|c|}{ C'est de la Cilicie jusqu'à Athènes que « l'essor... } & \multicolumn{2}{|l|}{ Alexis? } \\
\hline 9 & $751 C 1$ & Nom & I & & \multicolumn{2}{|l|}{ Solon, frg.25 (West) } & $2 \mathrm{~V}$ \\
\hline \multicolumn{8}{|c|}{ [prendre Solon] comme type de l'amant « tant que... » } \\
\hline 17 & 751E8 & Nom & NI & Cont & \multicolumn{2}{|l|}{ Solon, frg.26 (West) } & $2 \mathrm{~V}$ \\
\hline \multicolumn{6}{|c|}{ devenu vieux il s'exprime ainsi : « ... } & \multicolumn{2}{|l|}{ Abus. Commenté } \\
\hline 12 & 751D8 & Nom & NI & Déc & Sappho, frg.42 (Puech) & & $1 \mathrm{~V}$ \\
\hline \multicolumn{6}{|c|}{ Sappho s'adresse en ces termes : « ... } & \multicolumn{2}{|l|}{ Abus } \\
\hline
\end{tabular}




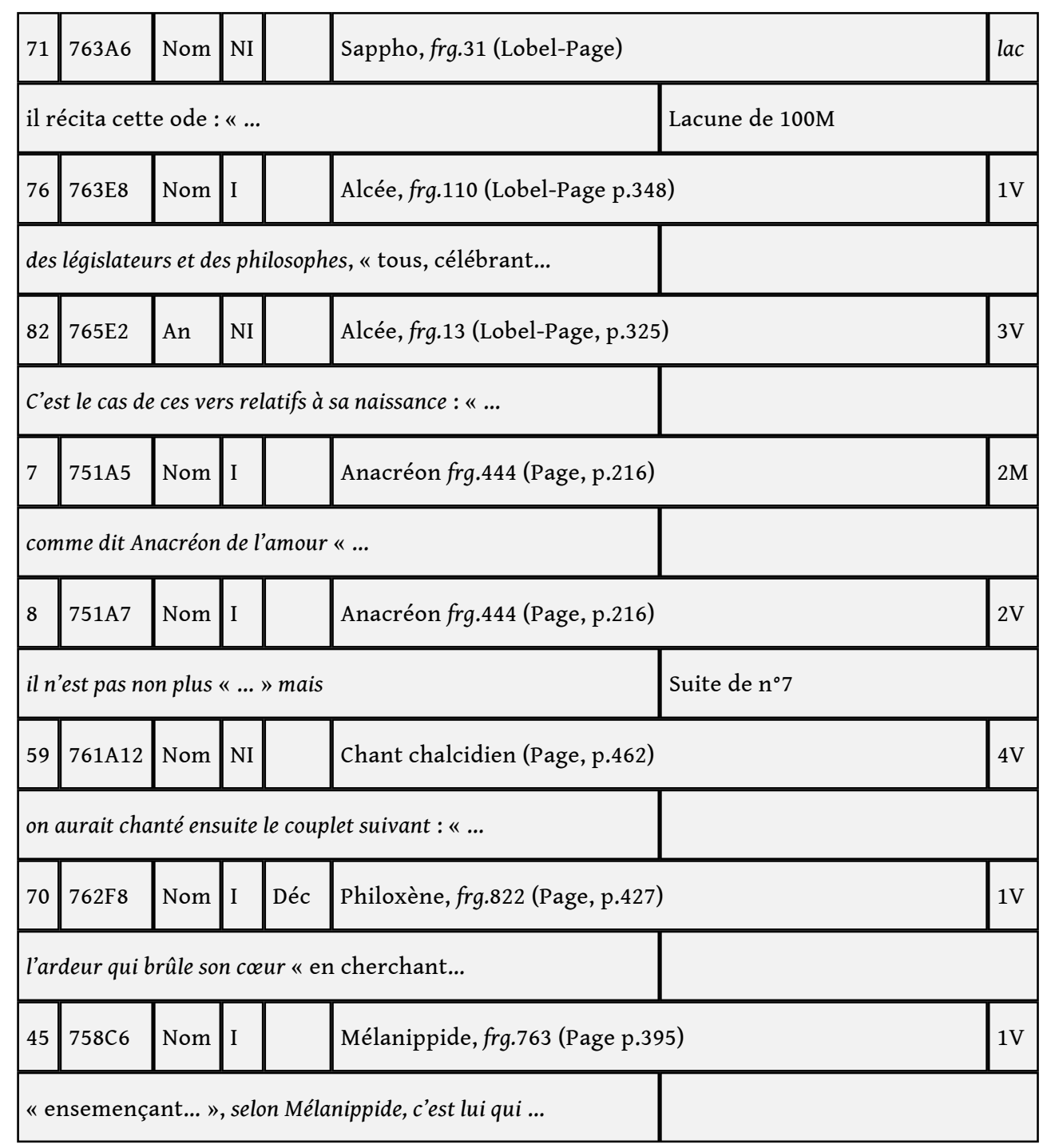

Platon (6)

\begin{tabular}{|c|c|c|c|c|c|c|c|}
\hline 14 & 751E1 & Nom & I & Déc & \multicolumn{2}{|l|}{ Platon Phèdre, 250 e } & $5 \mathrm{M}$ \\
\hline \multicolumn{6}{|c|}{ «...» selon Platon } & \multicolumn{2}{|l|}{ Citation libre } \\
\hline 16 & 751E5 & Nom & I & Déc & \multicolumn{2}{|l|}{ Platon, Lois, $8.839 \mathrm{~b}$} & $3 \mathrm{M}$ \\
\hline \multicolumn{8}{|c|}{ comme dit Platon « ... } \\
\hline 47 & 758F2 & An & I & Cont & \multicolumn{2}{|l|}{ Platon, Phèdre, 245 a } & $1 \mathrm{~L}$ \\
\hline \multicolumn{8}{|c|}{ « La troisième forme ... » pour } \\
\hline 79 & 764A8 & An & I & & \multicolumn{2}{|l|}{ Platon, Phèdre, $235 \mathrm{~b}$} & $4 \mathrm{M}$ \\
\hline \multicolumn{6}{|c|}{ tu n'as pas à nous les dire « même si nous t'en prions... } & Platon supra & \\
\hline
\end{tabular}




\begin{tabular}{|c|c|c|c|c|c|c|}
\hline 89 & 767D11 & Nom & I & Platon, République, $5.462 \mathrm{c}$ & & $5 \mathrm{M}$ \\
\hline \multicolumn{7}{|c|}{ dire, comme dans la cité de Platon : « ceci est à moi... } \\
\hline 90 & 767E1 & An & I & Platon, Lysis, 207 c & & $3 \mathrm{M}$ \\
\hline \multicolumn{5}{|c|}{ 'est pas d'emblée que " tout est commun entre amis" } & Pythagore & \\
\hline
\end{tabular}

\section{Autres philosophes (5)}

\begin{tabular}{|c|c|c|c|c|c|c|}
\hline 3 & 750D11 & Nom & I & \multicolumn{2}{|l|}{ Aristippe } & $2 \mathrm{~L}$ \\
\hline \multicolumn{5}{|c|}{ comme en témoigne la réponse d'Aristippe : « ... } & \multicolumn{2}{|l|}{ Style indirect. Comparaison } \\
\hline 37 & 757B5 & Nom & I & \multicolumn{2}{|l|}{ Chrysippe (von Arnim 2.1094) } & $1 \mathrm{M}$ \\
\hline \multicolumn{7}{|c|}{ il prétend qu'Arès signifie « le Tueur» } \\
\hline 87 & 767B3 & An & I & \multicolumn{2}{|l|}{ Chrysippe (von Arnim 3.718) } & $2 \mathrm{M}$ \\
\hline \multicolumn{7}{|c|}{ on dit que la beauté est « la fleur ... } \\
\hline 84 & 766F1 & Nom & I & \multicolumn{2}{|l|}{ Ariston (Von Arnim 1.390) } & $1 \mathrm{~L}$ \\
\hline \multicolumn{5}{|c|}{ de même que « ... », comme disait Ariston } & \multicolumn{2}{|l|}{ Style indirect } \\
\hline 52 & 759C9 & Nom & NI & Caton & & $2 \mathrm{~V}$ \\
\hline \multicolumn{5}{|c|}{ Le romain Caton disait que « ... } & \multicolumn{2}{|l|}{ Non littéral. Cf. Plut., Caton 9.8} \\
\hline
\end{tabular}

\section{BIBLIOGRAPHIE}

Babut Daniel, Plutarque et le stoïcisme, Paris : Les Belles Lettres, 1969.

Bompaire Jacques, Lucien écrivain. Imitation et création, Paris : E. de Boccard, 1958.

Bréchet Christophe, Homère dans l'œuvre de Plutarque. La référence homérique dans les Oeuvres Morales , thèse, Montpellier, 2003.

Bréchet Christophe, «Vers une philosophie de la citation poétique : écrit, oral et mémoire chez Plutarque ", Hermathena, 182, 2005, p. 101-134.

Compagnon Antoine, La seconde main ou le travail de la citation, Paris : Seuil, 1979.

Cribiore R., Writings, Teachers, and Students in Graeco-Roman Egypt, Atlanta, 1994. 
Darbo-Peschanski Catherine (ed), La citation dans l'antiquité, Grenoble : J. Millon, 2004.

Davidson, J.A., « Quotations and allusions in early Greek literature », Eranos, 53, 1955, p. 125-140.

Dorandi Tiziano, Le stylet et la tablette, Paris : Les Belles Lettres, L'âne d'or, 2000.

Elter A., De Gnomologiorum Graecorum historia atque origine, Leipzig : Teubner, 1893-1895.

Fairbanks A., « On Plutarch's Quotations from Early Greek Philosophers », Transactions of American Philological Association, 28, 1897, p. 75-87.

Flacelière Robert Plutarque traités 47-48 [Dialogue sur l'amour, Histoires d'amour], Paris : Les Belles Lettres, 1997 (1980).

Gangloff Anne, « Mentions et citations de poètes chez Dion Chrysostome », in : Nicolas 2006, p. 101-122.

Gotteland Sophie \& Oudot Estelle, Plutarque. Dialogue sur l'Amour. La philosophie de l'Antiquité. Paris : Flammarion, 2005.

Helmbold W.C. \& O'Neil E.N. : Plutarch's quotations, American philological association, Monographs $19,1969$.

Herschbell J. P., «Plutarch and stoicsm », «Plutarch and epicureanism », ANRW, II. 36.5, 1992, p. 3336-3382.

Horna, Konstanti, « Gnome, Gnomendichtung, Gnomologien », in : RE Pauly-Wissowa, sup. VI, 1935, col. 74-90.

Householder F. W., Literary quotation and allusion in Lucian, Columbia, 1941.

Howind Ed., De ratione citandi in ciceronis, Plutarchi, Senecae, Novi Testamenti scriptis obvia, (diss. Marburg), 1921.

Jacob Christian, «La citation dans les Deipnosophistes d'Athénée », in : Darbo-Peschanski 2004, p. 147-174.

Kullman W. \& Althof J. (ed.), Vermittlung und Tradierung von Wissen in der griechischen Kultur, Tübingen, 1993.

Lenfant, Dominique « Peut-on se fier aux 'fragments' d'historiens? L'exemple des citations d'Hérodote ", Ktèma 24, 1999, p. 103-121.

Martin H., « Plutarch's citation of Empedocles at Amatorius », G.R.B.S., 10, 1969 (a), p. 57-70.

Martin H., « Amatorius, 756 e-f : Plutarch's Citation of Parmenides and Hesiod », A.J.Ph., 1969 (b), p. $183-200$.

Nicolas Christian (ed.), Hôs ephat', dixerit quispiam, comme disait l'autre .... Mécanisme de la citation et de la mention dans les langue de l'Antiquité, Grenoble : Ellug, 2006.

Nicolas Christian, « Les contours linguistiques flous de la mention », in : Nicolas 2006, p. 125-145.

Pépin Jean, Mythes et allégorie, Paris : Aubier, 1958.

Rist John M. « Plutarch's "Amatorius": A Commentary on Plato's Theories of Love? », The Classical Quarterly, New Series, Vol. 51 (2), 2001, pp. 557-575

Rosier Laurence Le discours rapporté . Histoire, théories, pratiques, Duculot, 1998.

Stemplinger Eduard, Das Plagiat in der griechieschen Literatur, Leipzig-Berlin, 1912.

Van Den Hœk Annewies, « Techniques of quotation in Clement of Alexandria. A view of ancient literary working methods ", Vigiliae Christianae, 50 (3), 1996, p. 223-243 (Brill). 
Ziegler Konrad, « Plutarchos von Chaironea », RE, XXI 1, col. 636-962 [1949].

Ziegler Konrad, « Plagiat », RE, XXI 1, col.1956-1997 [1950].

\section{NOTES}

1. A propos des citations d'Hérodote par Athénée D. LENFANT écrit : «il s'agit parfois non d'une citation au sens moderne, mais d'une paraphrase abrégée; ou encore tel passage fait alterner citation, coupure et paraphrase, sans que jamais aucun indice ne permette de déceler la nature du traitement infligé à l'original » (1999 : 114-115).

2. Il s'agit bien, en effet, d'une maladie (!) : Plutarque est " hac consuetudine quasi morbo correptus » (HOWIND $1921: 24)$, comme les savants d'Athénée, vrais «obsédés de l'érudition » ( JACOB 2006 : 147).

3. "Quotations should be defined as having a considerable degree of literality. They need not to be verbatim in a modern sense, but they should follow the source to considerable extent. Paraphrase distinguishes itself from a quotation in that only a few words of the original source (sometimes only one or two) are present. Reminiscences, in turn, are different from allusions by having no literal correspondences but merely resemblance in theme or thought » (VAN DEN HOEK 1996:229). C'est moi qui souligne.

4. Voir Cicéron, de optimo genere oratorum, 14, et Jérôme, Lettres 57.4-5.

5. Voir, par exemple Lucien, Le pêcheur ou les ressuscités 1-4. Les duellistes enchaînent des citations d'Homère, puis d'Euripide quand l'un d'eux constate qu'Homère « ne lui sert plus de rien ».L'Eroticos logos propose un bref échange de ce type, entre adversaires, de citations de Solon (751 B-C).

6. : «En ce sens, la citation poétique a pleinement sa place dans l'askèsis ou la mélétè inhérente au mode de vie philosophique : elle est une de ces 'pratiques volontaires et personnelles destinées à opérer une transformation du moi' (Hadot). Avec une telle philosophie de la citation, on est également ou en même temps au cœur de la paideia, conçue comme culture et mode de vie, où l'on pense et parle naturellement en citant les poètes. » (BRÉCHET 2005 : 131-132).

7. Surtout Institution oratoire 9.2. Les analyses portant sur Platon (République 3) ou Aristote ( Rhétorique) sont plus rares.

8. Mais «tout le monde fait la preuve d'une assertion en avançant soit des exemples, soit des enthymèmes, et il n'y a rien en dehors de là » (Arist., Rhet. 1.2, 1356b4).

9. Le cadre agonistique de la sentence (et sa version ludique dans la joute citationnelle) est souligné par l'encouragement d'Aristote à « utiliser [les sentences] pour réfuter des dictons qui sont tombés dans le domaine public »(Rhet. 1395a20).

10. On en trouve un seul exemple dans le corpus de SPENGEL, Rhetores Graeci, Teubner, 1853-56.

11. Voir en particulier les Progymnasmata d'Hermogène ou de Théon (L. Spengel, Rhetores Graeci, Leipzig, 1853-56).

12. Comment il faut écrire l'histoire, 47.

13. Fronton (Lettres 2.9) écrit s'être préparé un recueil de citations de cinq livres, tirées de soixante ouvrages (excerpta ex libris sexaginta in quinque tomis), pour ses vieux jours. Marc-Aurèle

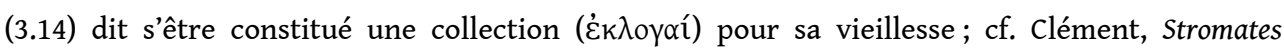
1.11.1: "Cet ouvrage n'est pas un écrit composé avec art pour être présenté, mais des notes stockées pour mon grand âge, un remède contre l'oubli, une simple réflexion, et une esquisse de ces propos nets et animés que j'ai eu le privilège d'entendre de gens bénis et véritablement remarquables".

14. Pline le Jeune (Lettres 3.5.10) raconte ainsi comment son oncle a pu écrire tant de livres malgré ses nombreuses occupations : "on lui lisait un écrit, il l'annotait et en extrayait des 
passages, car de toute lecture il tirait des extraits (adnotebat excerpebatque) ». Sur ce sujet voir le livre éclairant de T. DORANDI 2000.

15. Voir, par exemple, FAIRBANKS 1897, p. $84:$ «In general, it seems probable that Plutarch quotes Heracleitos, Xenophanes, and Parmenides commonly from an intermediate source ».

16. Voir STEMPLINGER 1912, p. 242 : «Les citations d'auteurs connus, surtout d'auteurs scolaires, provenaient généralement de la mémoire »; voir BRECHET 2005, p.101-105.

17. HELMHOLD \& O'NEIL 1959, p. IX : «Almost certainly Plutarch did not verify his quotations, or did so rarely, by looking up the passage in his texts ».

18. Voir BRÉCHET 2005. Cependant, il n'est pas sûr que la distance entre les deux procédures de citation (ex libro vs ex mente) soit si grande, comme le note C. ЈАСОВ $(2004$, p. 163) : «Or les arts de la mémoire antiques assimilent l'encodage mnémonique à une forme d'écriture sur des tablettes de cire ou une feuille de payprus mentale. Se remémorer, dès lors, c'est lire ce que l'on a inscrit dans sa mémoire ».

19. Lacune d'une centaine de lettres dans les manuscrits, correspondant à un fragment de Sappho (frg. 31 Lobel-Page).

20. Pour le détail, voir le répertoire de HELMBOLD-O'NEIL.

21. Cette limite est valable pour l'ensemble de l'œuvre de Plutarque: «Volkmannus [...] observavit eum nunquam plus 4 hexametris vel 5 trimetris continuis exscripsisse » (HowIND 1921: 24).

22. La différence entre citations textuelles et récritures (GANGLOFF 2006: 101) n'est pas pertinente ici.

23. Les chiffres suivants correspondent au nombre de colonnes de références par auteur. Parfois un passage de la source se trouve cité plusieurs fois dans l'œuvre.

24. Sur le détail des références plutarquéennes et leur répartition, voir Ziegler 1941: 715-728. Sur la fréquence relative des citations d'auteurs au II ${ }^{\mathrm{ème}}$ siècle et l'importance de l'usage scolaire, voir Gangloff 2006 : 104-106.

25. Pour une présentation précise du dialogue, voir GOTTELAND \& OUDOT 2005, et RIST 2001.

26. Même ici le dénombrement est inévitablement approximatif. Faut-il compter comme citation unique (ce que nous faisons ici) le passage "Homère appelle ce dieu 'assassin' et 'capricieux' » (757B3), qui relève deux épithètes n'apparaissant pas au même vers dans l'original (Iliade 5, vers 31 et 831)? D'autre part, nous n'avons pas comptabilisé des mentions qui ne semblent pas littérales, comme celle d'Aristote (l'historien ?) en 761A6, ou celle du poète Denys en 761B4: «à ce que rapporte le poète Denys dans ses Origines, Anton aurait été le nom de l'amant ».

27. Cf. Plutarque $607 \mathrm{C}-618 \mathrm{~B}$.

28. Def. orac. 415D (vers 1) ; Is. 355A et Quaest. 9.14.4 = 745A (vers 2-3).

29. Dans les Stromates de Clément le nom de Platon est mentionné dans $22 \%$ des citations, ceux d'Homère et Philon dans 34\%, et celui d'Euripide dans 52\% des cas (VAN DEN HÆK 1996 : 237).

30. Voir Howind $1921: 25-26$.

31. Howind (1921: 25), sur un échantillon de quinze traités de Plutarque, obtient un rapport légèrement inférieur : 210 mentions d'auteurs pour 549 citations (mais son travail est sévèrement critiqué par ZIEGLER 1949 : 928).

32. FAIRBANKS (1897: 79) note une seule erreur dans les attributions à Empédocle (fragment de Xénophane).

33. Subsistent de nombreux vers d'auteurs inconnus; voir par exemple, outres les (15) adespota tragiques et comiques (en particulier 766F7), le cas des (3) fragments épiques (attribués par Flacelière à Callimaque).

34. Cette assimilation est courante chez les écrivains; voir GANGLOFF 2006: 118 (à propos de Dion). 
35. «De certis atque receptis formulis inductoriis apud eum nihil fere dici potest, nam tam insigni varietate usus est, ut singulos quosque locos afferre deberem, si omnia accurate exponere vellem » (Howind $1921: 24$ ).

36. Le vers, ouvert pour la circonstance, autorise une intégration totale, soit en modifiant la désinence (cas ou forme verbale) d'un mot soit en insérant un terme. Voir, par exemple, 766 C 3 :

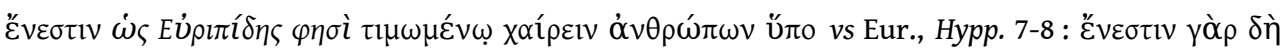

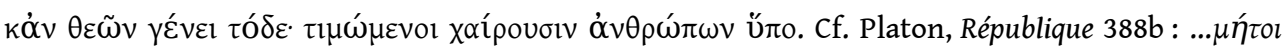

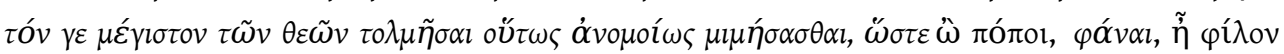

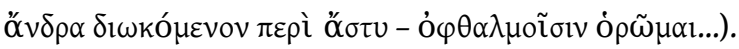

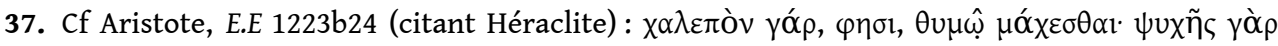
$\omega \dot{\omega} v \tilde{\imath} \tau \alpha$ l. Autres exemples dans FAIRBANKS 1897 : Héraclite frg. B $25=392 \mathrm{C}$ et 949A ; frg. B $29=604 \mathrm{~A}$ et 370B.

38. Voir néanmoins 756F ex Hésiode, Th. 120 (cf. Arist., Métaph. 963b) et 763D ex Xénophane frg. B 13.

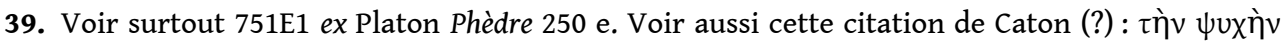

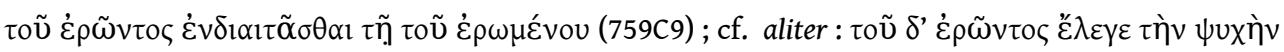

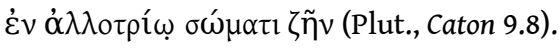

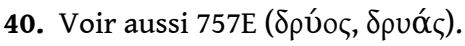

41. Pour un cas typique, dans un autre traité, voir Comment lire les poètes, à partir de $21 \mathrm{E}$, une série de sentences de Diogène suivie d'extraits tirés de Xénophon, puis d'Epicure.

42. Voir aussi en 769B-D, où les deux citations comiques semblent provenir du même auteur.

43. Empédocle, mentionné dans la séquence euripidéenne qui précède, fait lui-même déjà partie du contexte aristotélicien. MARTIN (1969a) a montré, en se fondant sur ce principe séquentiel, des emprunts évidents dans six traités plutarquéens à Aristote, Métaph., 983.

44. Dans l'Eroticos il s'agit de souligner la priorité et la primauté d'Eros sur Aphrodite alors que l'enjeu, dans le texte d'Aristote est de montrer qu' «il fallait que se trouva dans les êtres une cause capable de donner le mouvement et l'ordre aux choses ».

45. Voir Diogène-Laërce 7.181 : «Il était si prolixe qu'il écrivit plusieurs fois sur le même sujet, notait tout ce qui lui venait à l'esprit, se corrigeait souvent et usait abondamment de citations

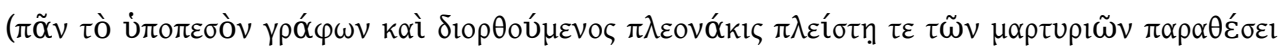
$\chi \rho \omega ́ \mu \varepsilon v o \varsigma)$. C'est au point qu'un jour, il cita la Médée d'Euripide presque en entier, et quelqu'un qui avait l'ouvrage en main, et à qui on demandait ce qu'il avait là, répondit: "La Médée de Chrysippe ».

46. Dans l'Eroticos toutefois on ne trouve aucune citation épicurienne, malgré le penchant épicurien de plusieurs interlocuteurs du dialogue (Protogène de Tarse, ami de Thespésios de Soles, un débauché [cf. Sur les délais de la justice divine, 563 B], Pemptidès de Thèbes, un sceptique à tendance épicurienne, et Zeuxippe de Lacédémone, connaisseur et sympathisant de l'épicurisme).

47. Voir Comment lire les poètes, 16E sq., 28A sq.

48. La traduction, comme ailleurs, est celle de R. Flacelière.

49. Cf. 761B4 vs 766C3. Voir aussi en 766F7-767A2 une citation comique à charge qui permet de dénoncer l'immoralité du personnage et de la thèse qu'il cautionne.

50. Voir aussi 763F4: «L'union de ces couples n'est nullement 'un joug immatériel...', selon l'expresion d'Euripide, elle est au contraire... ».

51. Voir aussi en $766 \mathrm{C} 3$ où une citation d'Euripide, prolongée, permet au discours d'aborder le thème de la rigueur de l'amour rejeté.

52. R. Flacelière juge bon d'isoler d'une parenthèse ce passage, emporté peut-être dans un lot de citations sur la vigne ou un groupement alphabétique sur le mot $\delta \rho v ́ o \varsigma$ (en cause dans le contexte). 
53. Voir le constat ambigu de A. GANGLOFf pour Dion (2006, p.114): Après avoir rappelé que « les trois fonctions que la rhétorique assigne traditionnellement aux exemples [sont] celles d'ornement, de modèle et de preuve », elle affirme simultanément que « la fonction ornementale des références mythico-poétiques joue certainement un rôle considérable dans l'éloquence de Dion » et que «sur l'ensemble des [377] références mythiques, on compte six cas seulement où l'exemple donné n'a pas d'autre fonction que celle d'ornement ".

54. Ainsi les deux vers redondants d'Eschyle que Daphné propose d'ajouter ( $\pi \rho o ́ \sigma \lambda \alpha \beta \varepsilon$ ) aux vers de Solon sur les cuisses (751C3), accentuent le ridicule de l'exaltation des cuisses masculines, et discrédite donc plus sûrement le témoin favorable que s'était choisi son adversaire, Protogène, pour conclure son plaidoyer de l'Eros garçonnier.

55. Voir aussi 751A : l'introduction du lemme « chasseur » qualifiant l'aigle chez Homère permet de filer le motif du parallèle entre chasse et langueur domestique.

56. Telle semble être la méprise de Plutarque qui fait d'Aphrodite le 'concepteur' d'Eros dans un fragment de Parménide où c'est en fait le $\delta \alpha i ́ \mu \omega v$ qu'il faut restituer (terme jamais appliqué à Aphrodite chez le philosophe) ; le détournement vient en fait de Platon (Conv.178B) et d'Aristote ( Méta.984b23) déjà, qui mésinterpètent cet extrait (MARTIN $1969 \mathrm{~b}$ :189-191).

57. De même Aristote, qui emprunte beaucoup de ses citations à Platon pour les philosophes présocratiques cite rarement exactement la lettre et se méprend sans doute en diverses occasions, parce qu'il consulte le texte dans un cadre secondaire, décontextualisé et critique.

58. Voir aussi DAVISON $1955: 132$.

59. Voir BRÉCHET 2005, p. 120-123.

60. Voir les remarques de A. Philippon au Comment lire les poètes : « Non seulement il déploie,

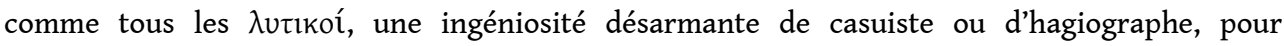
« innocenter » les poètes ou tirer une importante leçon morale de leurs vers les plus anodins, mais il commet sur plusieurs vers qu'il cite d'incontestables contresens, au point d'autoriser à croire qu'il les commente sans les avoir relus dans leur contexte " (Plutarque, CEuvres morales I.1, Paris : Belles Lettres, R. Flacelière et J. Irigoin (ed), 1987, p.70-71, et n.2).

61. Les nombreux cas de subversions figurant dans le traité hautement pédagogique Comment lire les poètes, sont à ce titre exemplaire : en 16E Plutarque subvertit totalement le sens de Od. 11.471 sq., sans que l'on puisse imputer cet écart à une ignorance du contexte; en $21 \mathrm{~A}$, il interprète Pindare, Isth. 4.48 comme une apologie de la violence alors qu'il s'agit d'une simple définition du pancrace (voir aussi 32F, sur Aristote, etc.).

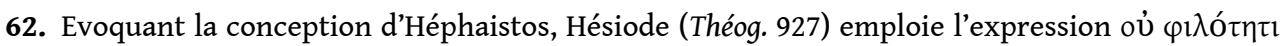
$\mu \imath ү \varepsilon \tilde{\imath} \sigma \alpha$.

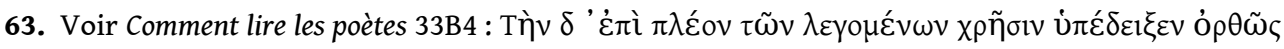

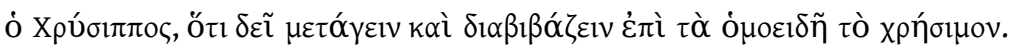

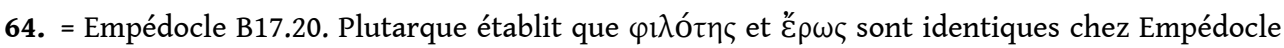
(cf. Quaest. conviv. 618B), mais en tronquant la citation (qui commence par « et la funeste haine séparée d'eux les vaut bien partout... ») et en interprétant le texte "l'amitié, parmi les éléments

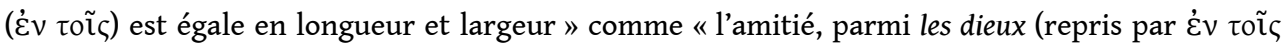

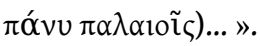

65. Voir e.g. 756F où le texte d'Hésiode est perverti (par l'omission de Gaia et Chaos qui sont cités avant Eros au commencement de tout), ou la manipulation des vers de Solon (751E7-8).

66. «Tu n'étais à mes yeux qu'une petite fille à qui la complaisance est encore étrangère

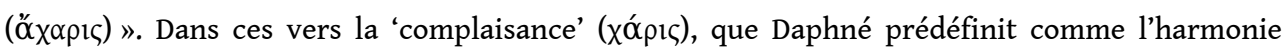
hétérosexuelle, signifie en fait -et presque au contraire dans la discussion - 'le plaisir sexuel'. Aux dépens de Sappho encore, voir aussi 762F8, où Plutarque prête à Sappho une passion qu'éprouve un Cyclope (et où il transforme le participe masculin en féminin...). 
67. I.e. 200 vs 98 ; pour un format identique: 22p. vs 23p [ed. Estienne], ou 55 vs 59 p. [ed. Philippon/Flacelière dans la CUF].

68. Est incluse dans cette liste un passage où Hésiode est précisément et nommément évoqué (756F1) sans que l'on ait pu relever une reprise littérale.

69. Une citation non intégrée est présentée (du type : « il dit que...).

\section{RÉSUMÉS}

Cet article étudie, dans l'Eroticos logos de Plutarque, un des textes plutarquéens les plus riches en citations, l'usage, l'assimilation et la fonction des citations, principalement poétiques. Il propose de distinguer différents modes d'intégration et de relation au discours propre, dans ce texte typique de la pratique plutarquéenne. La sélection et la distribution des citations révèlent une combinaison de différents procédés d'extraction et de manipulation, et une tactique plus discursive que démonstrative, qui dispose des citations comme un bricoleur ferait d'objets faiblement déterminés. L'hypothèse classique qui donne à la citation une valeur ornementale (et considère le plaisir du lecteur comme une fin et non comme une condition de lisibilité et un outil de persuasion) témoigne d'un profond malentendu sur la portée réelle de l'usage citationnel antique.

This paper focuses on the use, the syntaxic and logical integration and the various functions of the quotations in the Plutarch's Dialogue On love, which is especially rich in literary, mainly poetical, quotations. After general remarks on the culture-wide practice of quoting, we discriminate different levels of incorporation of alien ideas and sentences in this text typical of the plutarchean manner. Considering the selection and disposition of quotations, which includes contextomy, it appears that the way of extractiong and patching along and together the quotations favours not only rhetorical purpose but also dynamic effect. The classical and indeed antic conception of the esthetic function of the quotations and references comes short and fails to express the intellectual extent and impact of the quotational practice.

\section{INDEX}

Mots-clés : amour, citation, création, Plutarque, rhétorique 